\title{
Body Integrity Identity Disorder (BIID) - Ist der Amputationswunsch eine autonome Entscheidung oder Ausdruck einer neurologischen Störung?
}

Sabine Müller

\section{Einleitung}

Weltweit gibt es einige Tausend Menschen, die von Chirurgen die Amputation gesunder Gliedmaßen oder die Beibringung einer Querschnittslähmung fordern. Manche dieser Menschen verstümmeln sich selbst, um sich der unerwünschten Gliedmaßen zu entledigen.

Im Jahr 2000 ging die Nachricht durch die Presse, dass der schottische Chirurg Robert Smith zwei psychisch gestörten Männern jeweils ein gesundes Bein oberhalb des Knies amputiert habe. ${ }^{1}$ Aufsehen erregten diese beiden Fälle aus den Jahren 1997 und 1999 erst, als Smith 1999 einem dritten Patienten ein gesundes Bein amputieren wollte, was allerdings der neue Vorsitzende der Krankenhausbetreibergesellschaft nach einer Stellungnahme des Ethikkomitees untersagte. Smith rechtfertigte die Amputationen damit, dass diese Patienten ein gestörtes Körperbild hätten, ein Bein als überflüssig empfänden und lange Zeit darunter gelitten hätten. ${ }^{2}$ Durch eine BBC-Reportage im Jahr 2000 erreichte das Anliegen der Amputationswilligen einige Feuilletons, z. B. von The Independent ${ }^{3}$ sowie der Süddeutschen Zeitung, ${ }^{4}$ und löste eine Diskussion unter Medizinethikern aus. ${ }^{5}$

\footnotetext{
BBC 2, Horizon: „Complete Obsession - Body Dysmorhphia“, 17.02.2000. Vgl. Beckford-Ball (2000).

2 Vgl. Dyer (2000); Johnston/Elliot (2002); Fisher/Smith (2000); Smith/Fisher (2003).

3 Vgl. The Independent, 01.02.2000.

Vgl. Kissler (2005 a und b).

5 Vgl. Bayne/Levy (2005); Bridy (2004); Dudzinski (2005); Johnston/Elliot (2002); Tomasini (2006).
} 
Vor allem das Internet dient als Diskussionsplattform über die medizinisch nicht indizierten Wunschamputationen (elective amputations). Durch das Internet haben Betroffene, die lange glaubten, der einzige Mensch auf der Welt mit diesem seltsamen Wunsch zu sein, ${ }^{6}$ aus der Isolation und Anonymität herausgefunden und sich in Communities organisiert. In Anlehnung an Transsexuelle beschreiben sie sich als im falschen Körper gefangen: nämlich in einem Körper mit vier vollständigen Gliedmaßen, obwohl ihre wahre Identität die eines Amputierten sei. Die Interessenvertreter dieser Gruppen verteidigen die Amputationswünsche als autonome Entscheidungen, für die sie medizinische Hilfe und deren Finanzierung fordern; auch darin folgen sie den Transsexuellen, die in verschiedenen Staaten ihre Anliegen weitgehend erfolgreich durchgesetzt haben.?

Einschlägige Internetforen üben einen normalisierenden und wertschätzenden Effekt auf die normalerweise als pathologisch geltenden Vorstellungen aus. ${ }^{8}$ In Internetforen finden Amputationswillige Gleichgesinnte und werden Teil einer Gemeinschaft, die sich durch den Amputationswunsch definiert und sich als diskriminierte, aber selbstbestimmte Minderheit betrachtet. Diese Internetforen können die zwanghafte Fixierung auf den Amputationswunsch noch verstärken wie Suizid- oder Anorexie-Foren. Da sie außerdem Anleitungen für Selbstamputationen bereitstellen, ${ }^{9}$ können sie für vulnerable Individuen durchaus gefährlich sein. ${ }^{10}$

Bei den „freiwilligen Amputationen“ geht es nicht darum, Versicherungsleistungen zu erschleichen oder sich dem Armeedienst zu entziehen. Die Betreffenden haben keinerlei rationale Motive für die Selbstverstümmelung. ${ }^{11}$ Der Amputationswunsch resultiert auch nicht aus einem chronischen lokalen Schmerzsyndrom oder aus Phantomschmerzen in einem Amputationsstumpf. ${ }^{12}$ Die Begründung für den Wunsch nach Amputation eines bestimmten Körperteils beschränkt sich darauf, dass sie diesen als nicht zum Körper zugehörig fühlen, ihn vielmehr als störend empfinden, obwohl er nicht missgestaltet, gelähmt oder gefühllos ist.

Unter Ärzten und Psychologen herrscht Uneinigkeit über die Ursachen dieser Störung und deren Einordnung bzw. Abgrenzung von anderen Krankheitsbildern. Ob der Störung eine neurologische Ursache zugrunde liegt oder ob sie psychogen ist, ist noch unbekannt. Diese Kontroverse spiegelt sich in der Uneinigkeit hinsichtlich der Bezeichnung dieser Störung wider. In konkurrie-

6 Vgl. First (2004), S. 9.

7 Vgl. Steinmetzer/Groß (2007).

8 Vgl. Berger et al. (2005), S. 383.

9 Vgl. Berger et al. (2005), S. 383.

$10 \mathrm{Vgl}$. Wise/Kalyanam (2000), S. 343.

11 Vgl. Sorene et al. (2006), S. 594. - Dass Versicherungsbetrug nicht das leitende Motiv ist, zeigt sich darin, dass sie gegenüber Ärzten ihren Amputationswunsch meist nicht leugnen. Trotzdem beziehen einige ungerechtfertigt Sozialleistungen und Versicherungsprämien, z. B. der von Berger et al. (2005) beschriebene Patient (vgl. Fall 4).

12 Nach Amputationen haben viele Patienten Phantomschmerzen. Zu deren Therapie wird in manchen Fällen der Stumpf immer weiter amputiert, unter der Annahme, die Phantomschmerzen hätten ihre Ursache in beschädigten Nervenenden im Stumpf. Die scheibchenweise Amputation verschlimmert aber meist die Phantomschmerzen. Ramachandran hat nachgewiesen, dass Phantomschmerzen nicht lokal, sondern zentral verursacht werden. - Vgl. Ramachandran/Blakeslee (2002), S. 57-119. 
rendem Gebrauch sind die Begriffe Apotemnophilie, Body (Integrity) Identity Disorder (BIID bzw. BID) und Amputee Identity Disorder (AID). ${ }^{13}$ Obwohl es bei schweren psychotischen Störungen unter Umständen zu Selbstverletzungen bis hin zu Selbstamputationen kommt - besonders bei wahnhaften Schuldgefühlen oder religiösem Wahn ${ }^{14}$ - werden BIID-Patienten von Psychiatern als nicht psychotisch eingestuft. Psychosen stellen vielmehr ein Ausschlusskriterium für die BIID-Diagnose dar. ${ }^{15}$ Es wird kontrovers diskutiert, ob BIID eine neurologische Störung, eine Zwangsstörung, eine Körperbildstörung oder eine Identitätsstörung wie Transsexualität darstellt. Aufgrund der theoretischen Unklarheit besteht auch Uneinigkeit über die richtige Therapie. Tatsächlich gibt es bisher keine erfolgreiche Behandlungsmethode.

Unter Medizinethikern ist das Thema bisher wenig, aber sehr kontrovers diskutiert worden. Die australischen Medizinethiker Tim Bayne und Neil Levy halten - wie viele BIID-Patienten - die Amputationswünsche für autonome Entscheidungen. Sie fordern, die gewünschten Operationen zu erlauben, da diese die einzig wirksame Therapie und immer noch besser als selbst durchgeführte Amputationsversuche seien.$^{16}$ Noch weiter geht die amerikanische Medizinethikerin Annemarie Bridy (2004): Sie hält Wunsch-Amputationen für genauso legitim wie die heutzutage in den USA und Großbritannien allgemein akzeptierten kosmetischen Operationen. Und auch der britische Philosoph Floris Tomasini (2006) fordert, dass Chirurgen Amputationen bei BIIDPatienten durchführen; er übernimmt die Position der BIID-Vertreter sowie des Chirurgen Smith kritiklos und ignoriert die Publikationen von Psychiatern und Neurologen zu BIID.

Auf der anderen Seite haben sich einige Medizinethiker, Ärzte und Politiker vehement gegen die von Smith durchgeführten und propagierten Amputationen gesunder Gliedmaßen gewandt. ${ }^{17}$

Neueste Forschungsergebnisse, insbesondere der Neurologen Vilayanur Ramachandran und Paul McGeoch (2007), legen nahe, dass BIID und damit der Amputationswunsch die Folge einer hirnorganischen Störung sind und daher nicht als Ausdruck des freien Willens einer autonomen Person gewertet werden dürfen. Statt Amputationen wäre dann eine Therapie des Gehirns indiziert. Dies würde allerdings bei vielen BIID-Patienten auf heftigen Widerstand stoßen, da sie ihren Wunsch nicht für eine hirnorganische Störung halten, sondern für eine bewusste Entscheidung bzw. für einen Bestandteil ihrer Identität. Eine mangelnde Krankheitseinsicht ist aber wahrscheinlich Teil dieses Störungsbildes und auch hirnorganisch bedingt, ähnlich wie bei Körperbild-

13 Siehe hierzu Kapitel 2.

14 Vgl. Berger et al. (2005), S. 380. - Die Selbstverstümmelung (Automutilation) der Genitalien wird auch von manchen religiösen Gruppen praktiziert, z. B. in der Antike von Priestern der Kybele sowie im heutigen Indien von Angehörigen der hinduistischen Sekte Hirja. - http://de.wikipedia.org/Selbstverstümmelung.

15 Vgl. Sorene et al. (2006), S. 594. - Auch die 52 von First (2004) interviewten BIID-Patienten waren nicht psychotisch.

16 Vgl. Bayne/Levy (2005).

17 Z. B. Arthur Caplan (zit. nach Dotinga [2000]), Loewy (http://lists.ruhr-uni-bochum.de/mailman/listinfo/ medethik-list), der schottische Parlamentarier Canavan (vgl. Bayne/Levy [2005], S. 85), Beckford-Ball (2000), Johnston/Elliott (2002), Munro (2000), Dyer (2000) und Kissler (2005 a und b). 
störungen nach Schlaganfällen und bei Anorexie. Möglicherweise kann die funktionelle Bildgebung nicht nur bei der Lokalisierung des Krankheitsherds helfen, sondern auch zur Krankheitseinsicht und Compliance der Patienten beitragen. Dieser Weg ist gewiss schwieriger als Amputationen, doch sein Ziel ist ein Mensch mit einem unversehrten Körper in Übereinstimmung mit seinem Körperbild statt eines dauerhaft schwer behinderten Menschen.

\section{Definition der Störung}

Seit ca. 1880 wird in medizinischen Schriften das Phänomen beschrieben, dass einige Menschen sich sexuell von Amputierten angezogen fühlen. ${ }^{18}$ Money et al. (1977) haben dafür den Begriff Apotemnophilie ${ }^{19}$ eingeführt, also wörtlich Liebe zum Abschneiden, um ein Syndrom zu beschreiben, bei dem eine körperlich nicht behinderte Person mit dem Wunsch beschäftigt ist, einen gesunden Körperteil amputiert zu bekommen, was durch eine sexuelle Erregung durch Amputierte motiviert sein soll. Money et al. (1977) interpretieren Apotemnophilie als eine Paraphilie. ${ }^{20}$ Wie die meisten Paraphilien kommt auch Apotemnophilie vorwiegend bei Männern vor ${ }^{21}$ sie könnte auch mit (abgelehnter) Homosexualität zusammenhängen. ${ }^{22}$ Der Begriff Apotemnophilie wird heute sowohl zur Bezeichnung eines medizinisch nicht indizierten Amputationswunsches als auch im Sinne von Amputationsfetischismus verwendet, ${ }^{23}$ da beide häufig zusammenhängen.

Andere Bezeichnungen für den Wunsch nach der Amputation gesunder Körperteile sind Amputee Identity Disorder und Body Integrity Identity Disorder (BIID). Letztere wird zunehmend verwendet, wenn auch weniger in der Fachliteratur. Er stellt im Gegensatz zum Begriff Apotemnophilie das Syndrom nicht mehr in den Kontext einer Paraphilie, ${ }^{24}$ sondern betont den Aspekt der Identitätsstörung.

Derzeit ist umstritten, ob BIID ein eigenständiges Krankheitsbild ist, für das eine neue diagnostische Definition nach DSM erforderlich ist, oder ob es unter andere psychiatrische Störungen zu subsumieren ist, insbesondere unter Zwangsstörungen (Obsessive-Compulsive Disorder, OCD) oder unter Body

18 Vgl. Bruno (1997), S. 244; Everaerd (1983), S. 285.

19 Aus dem Griechischen: $a p o=$ von; temnein = schneiden, philia = Liebe. - Money/Jobaris/Furth (1977), S. 115.

20 Paraphilien (von griech: pará = abseits, neben, und philía = Liebe) sind nach dem aktuellen DSM-Handbuch der American Psychiatric Association (DSM-IV-TR) psychische Störungen, die als von der Norm abweichende, ausgeprägte, wiederkehrende sexuelle Phantasien, Bedürfnisse oder Verhaltensweisen auftreten und sich auf unbelebte Objekte, auf Leiden oder Demütigung und/oder auf nicht einverständnisfähige Personen beziehen und in klinisch relevantem Maß Leiden oder Beeinträchtigung in sozialen, beruflichen oder anderen wichtigen Funktionsbereichen bei der betroffenen Person und/oder ihren Opfern hervorrufen. Häufige Paraphilien sind Fetischismus, Fetischistischer Transvestismus, Exhibitionismus, Voyeurismus, Pädophilie, Sadomasochismus, Frotteurismus, Zoophilie, Nekrophilie, Acroptomophilie, Apotemnophilie. - Ausschlusskriterien für eine Paraphilie sind: Verursachung dieser Störung durch akute Intoxikation, Manie, Schizophrenie, geistige Behinderung, Demenz, Persönlichkeitsveränderung oder eine medizinische Krankheit. - Vgl. American Psychiatric Association (2000).

21 Vgl. Braam et al. (2006), S. 33. - In der Studie von First (2004) waren von den 52 BIID-Patienten 47 Männer, 4 Frauen und ein Intersexueller („,born intersexed, raised as a male and then reassigned to female“, S. 3).

22 Vgl. Money/Jobaris/Furth (1977); Berger et al. (2005); Evereard (1983), S. 287; First (2004), S. 3.

23 Vgl. Wise/Kalyanam (2000), S. 339.

24 Vgl. Braam et al. (2006), S. $32 \mathrm{f}$. 
Dysmorphic Disorder (BDD). ${ }^{25}$ Diskutiert wird, insbesondere von den Betroffenen selbst, ob BIID mit Transsexualität bzw. Gender Identity Disorder (GID) verwandt sei. Dafür könnte sprechen, dass BIID-Patienten wie Transsexuelle eine Identität zu haben glauben, die der physischen Identität entgegengesetzt ist, jahrelang die Wunschidentität simulieren und hoffen, durch eine Amputation das äußere Körperbild mit dem inneren in Übereinstimmung zu bringen. Den Frauenkleidern, Frauenschuhen, Perücken etc. der Mann-zu-Frau-Transsexuellen entsprechen Krücken und Rollstühle der BIID-Patienten; der Penis- und Hodenamputation die Amputation von Armen oder Beinen. Es gibt tatsächlich auffallend viele Mann-zu-Frau-Transsexuelle unter den BIID-Patienten. ${ }^{26}$

Nach einer Klassifikation von Richard Bruno (1997) sind drei Typen von Apotemnophilen zu unterscheiden: erstens die Wannabes (Would-be amputees), die sich nach einer bestimmten körperlichen Behinderung sehnen, möglichst durch Amputation; zweitens die Pretenders, die sich so verhalten, als seien sie körperlich behindert, und dazu Hilfsmittel verwenden, z. B. Rollstühle, Krücken und Bandagen zum Abbinden von Gliedmaßen; drittens die Devotees, die sich sexuell von körperbehinderten, besonders von amputierten Personen angezogen fühlen. ${ }^{27}$

\section{Fallbeispiele}

\section{Fall: Apotemnophilie maskiert als medizinische Morbidität}

Mike Bensler und Douglas Paauw sowie S. Storm und Michael D. Weiss am Washington Medical Center berichten über einen 24-jährigen Maler, der mit Schwäche und Taubheitsgefühlen in den Füßen in der neurologischen Abteilung aufgenommen wurde. ${ }^{28}$ Es fiel auf, dass seine distalen Zehenglieder fehlten, was der Patient auf Erfrierungen bei einem Bergsteigeunfall zurückführte. Da die Nervenleitfähigkeit verändert war und an beiden Beinen Cellulitis vorlag, wurde Polyradikulitis (Guillian-Barré-Syndrom) diagnostiziert; der Patient erhielt fünf Tage lang intravenös Immunoglobulin. Wenige Tage nach seiner Entlassung erschien er in der Notaufnahme mit Schmerzen, Schwächegefühl und Schwellungen in den Beinen sowie hohem Fieber und beschleunigtem Herzschlag. Der Patient erhielt über mehrere Tage intravenös Antibiotika, zeigte jedoch keine Besserung. Auf Anraten eines Chirurgen sollten bilaterale Einschnitte und Drainagen gesetzt werden, um eine nekrotisierende Fasziitis zu verhindern. Der Patient bat den Chirurgen aber um beidseitige Amputationen unterhalb der Knie. Dies schien dem hinzugezogenen orthopädischen Chi-

\footnotetext{
25 Vgl. Braam et al. (2006), S. 32 und $36 \mathrm{f}$.

26 Vgl. Money/Jobaris/Furth (1977); Berger et al. (2005); Evereard (1983), S. 287; First (2004), S. 3; Braam et al. (2006).

27 Vgl. Braam et al. (2006), S. 32 f., und Bruno (1997), S. 243. - Die Devotees werden auch als Allo-Apotemnophile bzw. Acrotomophile (acros = Extremität, tome $=$ Schnitt) bezeichnet.

Es gibt zahlreiche Internetseiten mit Amputierten-Pornographie, und schon vor der Etablierung des Internets gab es einen Markt für derartige Pornographie. Hirschfeld hat bereits 1948 den Amputierten-Fetischismus beschrieben. Vgl. Bensler/Paauw (2003), S. 676; Wise/Kalyanam (2000), S. 339 und 342.

$28 \mathrm{Vgl}$. zu diesem Fall Bensler/Paauw (2003) und Storm/Weiss (2003).
} 
rurgen aber nicht indiziert, und der Patient erklärte sich mit den Inzisionen und Drainagen einverstanden. Eine Befragung der Ehefrau und der Mutter des Patienten ergab, dass dieser von Amputationen fasziniert sei, seine Zehenglieder mit Hilfe von Abschnürbinden selbst amputiert habe und immer wieder seine Beine abgeschnürt habe. Die dadurch erzeugte Blutleere hatte offenbar die beidseitige Neuropathie und Cellulitis erzeugt. Die weitere Anamnese ergab frühere Verletzungen an den Beinen, unter anderem eine selbst herbeigeführte Schussverletzung an einem Zeh. Es stellte sich heraus, dass sein biologischer Vater an paranoider Schizophrenie gelitten und zwei Jahre zuvor Suizid begangen hatte. Aufgrund der Anamnese wurde der Patient mit Verdacht auf Apotemnophilie in die Psychiatrie überwiesen. Nach zweiwöchigem stationärem Aufenthalt mit täglicher Psychotherapie wurde er entlassen - mit dem andauernden Wunsch nach Amputation seiner Unterschenkel, begründet mit der Überzeugung, dass seine Infektionen zurückkommen würden.

\section{Fall: Amputationswunsch bei einem Pretender}

Der Amsterdamer Psychiater Arjan Braam und seine Kollegen haben im Jahr 2006 den Fall eines 35-jährigen Mannes beschrieben, der mit dem Wunsch, sein linkes Bein 15 Zentimeter oberhalb des Knies amputiert zu bekommen, zu ihnen überwiesen worden war. ${ }^{29}$ Er hatte keine Erklärung für diesen Wunsch, außer dass sich dieses Bein völlig nutzlos anfühlte. Er verglich sein Problem mit dem von Transsexuellen. Die Rolle eines Behinderten zu haben, betrachtet der Patient als Gerechtigkeit gegenüber seinem wirklichen Selbst. Sein Amputationswunsch besteht schon seit dem 9. Lebensjahr, variiert kaum in der Intensität und ist nicht mit sexueller Erregung verbunden. In seiner Freizeit bindet der Mann das linke Bein mit Bandagen hoch und verwendet Krücken oder einen Rollstuhl zur Fortbewegung. Dies praktiziert er so oft wie möglich, seit einigen Jahren auch in Gegenwart seiner Frau sowie im Urlaub. Er glaubt, er würde im Alltag besser funktionieren und könne sich besser konzentrieren und sogar noch eine neue Ausbildung, ehrenamtliche Tätigkeiten oder ein neues Hobby beginnen, wenn er immer mit Krücken herumlaufen dürfte. Bis auf die Vorstellung, sein linkes Bein sei völlig überflüssig, und das Gefühl der Depersonalisierung in Bezug auf dieses Bein erscheint er psychisch normal, abgesehen von einer gewissen Ängstlichkeit und sozialen Isolation. Hinweise auf eine Persönlichkeitsstörung ließen sich nicht finden. Die körperlichen, neurologischen Untersuchungen sowie eine strukturelle MRT-Untersuchung zeigten regelrechte Befunde. Braam et al. diagnostizierten Apotemnophilie. Sie versuchten eine Therapie mit dem angstlösenden Medikament Oxazepam, später mit dem Serotoninwiederaufnahmehemmer Paroxetin, was zwar gegen die Unruhe, das frühzeitige morgendliche Aufwachen und die Dysphorie half, aber den Amputationswunsch in keiner Weise abschwächte. Auch die Therapie bei mehreren einschlägig erfahrenen Psychotherapeuten war erfolglos. Braam und seine Kollegen probierten anschließend eine modifizierte kognitive Verhaltenstherapie für Zwangsstörungen. Sie hatten festgestellt, dass das

29 Vgl. Braam et al. (2006). 
Pretender-Verhalten zwar eine Zeitlang das Leiden linderte, langfristig aber die Beschäftigung mit dem Amputationswunsch verstärkte und dazu führte, dass andere Beschäftigungen vernachlässigt wurden und deswegen sogar mit der Ehefrau ein Verzicht auf Kinder vereinbart worden war. Als Ziel vereinbarte der Therapeut mit dem Patienten die Verminderung des Leidens durch das ständige Nachsinnen über die Amputation, aber nicht die Vorbereitung auf eine Amputation; dies zu akzeptieren bereitete dem Patienten einige Mühe, zumal er betonte, dass sein Problem weniger mit einer Zwangsstörung als mit Transsexualität gemein habe. Es stellte sich heraus, dass die Beschäftigung mit dem Amputationswunsch durch Ruhe und Entspannung verstärkt wurde und durch intensive Arbeit oder durch das Hören seiner Lieblingsmusik zurückgedrängt werden konnte. Die kognitive Verhaltenstherapie erreichte zwar eine Reduzierung des Pretender-Verhaltens und eine gewisse Verdrängung der Zwangsgedanken durch geeignete Ablenkungsstrategien, aber der Patient gelangte nicht zur Krankheitseinsicht. Auch wenn er nun weniger unter den negativen Begleiterscheinungen litt, war er nach über 16-monatiger Therapie nicht bereit, sich ein Leben ohne Amputation vorzustellen. Vielmehr nahm seine Identifikation damit noch zu, da er sich nun traute, Verwandten und Arbeitskollegen davon zu berichten, und weniger negative Reaktionen erhielt, als er gedacht hatte. Braam et al. stellen fest, dass bei dem Patienten nicht nur eine Zwangsstörung und eine Body Dysmorphic Disorder vorliege, sondern auch eine Identitätsstörung.

\section{Fall: Amputierten-Fetischismus und Penis-Selbstamputation}

Thomas N. Wise und Ram Chandran Kalyanam von der Abteilung für Psychiatrie und Verhaltenswissenschaft der John Hopkins University School of Medicine in Baltimore, beschreiben den Fall eines 49-jährigen Mannes, der seinen Penis gemäß einer Anleitung aus dem Internet amputiert hatte. ${ }^{30}$ Obwohl er steriles, professionelles Operationsbesteck verwendet und seinen Penis vor dem Abschneiden abgeschnürt hatte, erlitt er starke Schmerzen und konnte die Blutung nicht stoppen; daher rief er eine Ambulanz. Im Krankenhaus wurde sein Penis wieder angenäht; eine Erektion war danach allerdings nicht mehr möglich. Die Anamnese ergab, dass der Mann bereits einige Wochen vor seiner Selbstamputation versucht hatte, seinen Penis durch Abschnüren zum Absterben zu bringen, und dass er seit einem halben Jahr davon phantasierte, seinen Penis abzuschneiden, und dabei masturbierte. Zeichen von Transsexualität, Transvestismus und Homosexualität zeigte der Patient nicht, aber er war seit seiner Pubertät fetischistisch auf amputierte Frauen fixiert. Er hatte auch eine Frau mit einem amputierten Bein geheiratet, die allerdings sehr erbost über seinen Fetischismus war. Zu der Zeit, als der Mann den Amputationswunsch entwickelte, war er in verschiedener Hinsicht belastet: geschieden, im Streit mit seiner Exfrau um das Besuchsrecht für seinen Sohn und Unterhaltszahlungen, belastet durch die Erkrankung seiner Mutter, die künstlich beatmet werden musste, sowie durch berufliche und finanzielle Probleme. Er litt

30 Vgl. Wise/Kalyanam (2000). 
in dieser Zeit unter depressiver Stimmung, Schlafstörungen und verringertem Antrieb. Er war aber weder psychotisch, noch konsumierte er Drogen. Während seines Klinikaufenthalts war er über die Penisamputation sehr beschämt und bereute diese. Er wurde erst stationär, dann ambulant mit Antidepressiva sowie Psychotherapie behandelt, wobei sein Fetischismus fortbestand, aber keine erneuten Amputationswünsche auftraten. Wise und Kalyanam diskutieren, ob die Penisamputation aus dem Amputiertenfetischismus durch die äußeren Belastungen und die Depression hervorgegangen sei.

\section{Fall: Selbstamputationen bei einem Mann-zu-Frau-Transsexuellen}

Bertrand Berger et al. (2005) haben einen transsexuellen Mann mit Amputationswunsch beschrieben, den sie als nicht psychotisch und nicht paraphil einstufen. Dieser Patient glaubte als Kind, dass er, wenn er behindert wäre, von seinen Eltern geliebt würde, und dass seine Mutter lieber ein Mädchen gehabt hätte. Der Patient ging zum Militär, wurde aber wegen Diabetes und Asthma ausgemustert, und wurde dann Manager in einem Prothesenlabor. Er heiratete und zeugte sechs Kinder. Seine Frau ließ sich scheiden, als sie feststellte, dass er heimlich Frauenkleider trug und einen Amputierten simulierte. Im Alter von 51 Jahren begann er, weibliche Hormone einzunehmen, und lebte vier Jahre lang als Frau. Kurz nach der Scheidung unternahm er einen Versuch, sein linkes Bein zu amputieren, was allerdings misslang. Er genoss es, in der Heilungsphase einen Rollstuhl zu benutzen, weil er sich darin ,authentischer" und „femininer" fühlte. Einige Monate nach der Scheidung unternahm er einen zweiten Amputationsversuch, diesmal an beiden Beinen, die er sieben Stunden lang in Trockeneis packte. Damit erreichte er, dass ein Chirurg beide Beine oberhalb der Knie amputierte. Mit den Amputationen erklärte er sich sehr zufrieden: Er fühle sich nun als eine vollständige Person. Zwei bis drei Jahre nach seinen Amputationen wünschte er nicht mehr, eine Frau zu sein, und lebte wieder als Mann. Er hielt seine Transsexualität nun für eine Maskerade und Symptomverschiebung:

„The amputation desire came first and foremost. It was just that an avenue became available to do something about one of my obsessions [male-to-female transsexual] through ,approved channels'. Both were, as I now understand them, defense postures I took as a way to absent myself from situations in which I felt incompetent or threatened. One can fully transform oneself in becoming an amputee. The adoption of the desired characteristic is complete and without ambiguity. No matter how many hormones or other drugs I took or however much surgery I endured, I, as a biological male, could never really be a female, as I understand that label. Once I was an amputee, the thin shell that was my feminine presentation was seen more and more for the masquerade it was. ${ }^{\text {“31 }}$

Der Patient beendete die Kontakte zur Wannabe Community und nahm stattdessen Verbindungen zur ,legitimen“ Amputiertengemeinde auf. Dass seine Amputationen selbstverschuldet waren, verschwieg er nicht nur dort, sondern

31 Berger et al. (2005), S. 382. 
auch gegenüber der Veteranenverwaltung, von der er nun eine hundertprozentige Schwerbehindertenrente erhielt. Eine Freundschaft zu einem an allen vier Gliedmaßen amputierten Mann löste Gedanken an eine Armamputation aus. Diese führten zu einer Einweisung in die Psychiatrie, wo er hoch dosiertes Fluoxetin erhielt. Diese Therapie verminderte die Intensität seines erneuten Amputationswunsches etwas, aber nicht vollständig.

\section{Erklärungsmodelle für den Amputationswunsch}

Was die Ursachen des Wunsches nach Amputation gesunder Gliedmaßen sind, ist umstritten. Es gibt verschiedene Erklärungsmodelle, doch keines scheint ihn umfassend erklären zu können. Die Unklarheit über die Ätiologie des Amputationswunsches hat Ratlosigkeit über eine geeignete Therapie zur Folge, und tatsächlich gibt es bis heute keine wirksame Behandlung.

\subsection{Psychologische Erklärungsmodelle}

\section{Paraphilie}

Money et al. (1977) beschreiben den Wunsch nach Amputation als eine Paraphilie: Apotemnophilie als „Liebe zur Amputation“, die in Zusammenhang mit der Erotisierung des Amputationsstumpfes stehe. Es handele sich dabei eher um eine fixe Idee als um einen paranoiden Wahn. Wie die meisten Paraphilien tritt auch Apotemnophilie hauptsächlich bei Männern auf, offenbar besonders bei Homosexuellen und Transsexuellen..$^{32}$ Möglicherweise dient sie der Kompensation abgelehnter Homosexualität oder der Abwendung einer Penisamputation bei Transsexualität. Nach dieser Erklärung sind der Amputationswunsch (Autoapotemnophilie) und der Amputationsfetischismus (Alloapotemnophilie) eng verbunden. Die überwiegende Mehrheit der von First (2004) befragten 52 BIID-Patienten, nämlich $87 \%$, fühlt sich sexuell von Amputierten angezogen. Darüber hinaus hat fast ein Drittel mindestens eine weitere Pa-

32 Vgl. Everaerd (1983), S. 287; Money/Jobaris/Furth (1977); First (2004), S. 3; Berger et al. (2005).

Die beiden von Money/Jobaris/Furth (1977) beschriebenen Männer sind bisexuell; einer davon bezeichnet sich als versteckt transsexuell. Beide haben erotische Phantasien bezüglich eigener Amputationen und fühlen sich von Amputierten sexuell angezogen. Beide Männer bekunden, dass sie mit einer Amputation ein „overachievement“ erreichen wollen, also ihre Leistungsfähigkeit trotz Amputation beweisen wollen, was sie sexuell erregend finden. Damit wollen sie ihre selbst in Frage gestellte männliche Identität stabilisieren, insbesondere gegenüber väterlicher Ablehnung und religiöser Verurteilung. Der eine betrachtet seinen Amputationswunsch als „an avenue to run away from homosexuality“ (S. 123). Der andere sagt: „I would rather this [amputation] than lose the penis which would mean that I would be like a woman. ... My entire erotic activity now consists of trying to make ,real' the fantasy that I am an amputated homosexual adolescent, for in possessing my stump, I can, concurrently, possess my penis." (S. 118). Die Amputation könnte also ein Weg sein, den Penis und die Maskulinität zu erhalten (S. 124). - Auch der von Berger et al. (2005) beschriebene Patient ist ein Mann-zu-Frau-Transsexueller. Bei diesem hat die Amputation beider Unterschenkel dazu geführt, dass er auf die Amputation seiner Genitalien verzichtet hat und wieder als Mann lebt. Er glaubt, dass seine Annahme, transsexuell zu sein, ein Ausweichen vor seiner Identität als Amputierter gewesen sei.

Der von Evereard (1983) beschriebene Patient ist ein Homosexueller, der seine sexuellen Neigungen weitgehend verdrängt hat. 
raphilie (Transvestitismus, Fußfetischismus, Lederfetischismus, Masochismus, Pädophilie).

Heute wird die Theorie, nach der Apotemnophilie im Wesentlichen eine Paraphilie sei, vielfach für veraltet gehalten. Anne Lawrence (2006) verteidigt sie aber und weist auf die Ähnlichkeit von nichthomosexueller Mannzu-Frau-Transsexualität (MtF-GID) und Apotemnophilie hin: Transsexualität und Apotemnophilie zeigen erstens eine starke männliche Prädominanz und zweitens eine Prävalenz anderer Paraphilien. Lawrence nimmt an, dass bei beiden ein erotic target location error vorliege: Wie nichthomosexuelle transsexuelle Männer durch die Vorstellung ihrer selbst als Frau sexuell erregt werden (Autogynephilie), so werden Personen mit BIID durch die Vorstellung ihrer selbst als Amputierte sexuell erregt (Apotemnophilie). Demnach könne Apotemnophilie die höchst seltene Kombination eines ungewöhnlichen erotic target (nämlich Amputierte) mit einem erotic target location error sein, also eine Kombination aus Acrotomophilie und Autoerotizismus.

\section{Factitiuos Disability Disorder}

Richard Bruno (1997) lehnt die Klassifikation von Apotemnophilie als eine Paraphilie ab, da nicht in allen Fällen eine sexuelle Komponente zu finden sei. Er hat stattdessen ein einheitliches psychologisches Erklärungsmodell vorgeschlagen, das Devotees, Pretenders und Wannabes mit Factitiuos Disorder (erkünstelte Störung, DSM-IV 300.19) in Zusammenhang bringt. Factitiuos Disorder ist eine psychische Störung, bei der eine Behinderung vorgetäuscht wird, um Aufmerksamkeit und Zuwendung zu erhalten. Sie ähnelt dem MünchhausenSyndrom. ${ }^{33}$ Bruno (1997) beschreibt zwei Patientinnen, die in der Kindheit extrem wenig Zuwendung erfahren hatten und glaubten, wenn sie eine Behinderung hätten, würden sie geliebt. Im ersten Fall, den Bruno als Devotee/ Pretender klassifiziert, strebt die Frau anfangs danach, mit einem behinderten Mann in der Öffentlichkeit gesehen zu werden; später verwendet sie selbst einen Rollstuhl, um Aufmerksamkeit und Zuwendung von fremden Menschen zu erfahren. Im zweiten Fall, den Bruno als Wannabe unaware klassifiziert, simuliert eine Frau eine Behinderung, die angeblich eine Spätfolge ihrer Kinderlähmung sein soll; auch ihr Verhalten dient vor allem dem Gewinn von Zuwendung, aber auch der Rechtfertigung einer Regression, insbesondere für ihren Rückzug aus dem Arbeitsleben. Brunos Erklärungsmodell definiert eine diagnostische Gruppe Factitiuos Disability Disorder, unter die er Devotees, Pretenders, Wannabes sowie Personen mit Factitiuos Disorder subsumiert. Die vier Typen unterscheiden sich nach Bruno nur gemäß zweier Kriterien: erstens dem

33 Das Münchhausen-Syndrom ist eine psychische Störung, die sich darin äußert, dass die Patienten körperliche Störungen erfinden bzw. durch Selbstverletzungen oder Selbstvergiftungen hervorrufen, um sie Ärzten zu präsentieren. (ICD-10: Code F68.1) Die Patienten verlangen meist aufwendige Diagnostik und Therapien, inklusive invasiver Eingriffe. Der Krankheitsgewinn liegt offenbar in der Zuwendung durch Ärzte und Pfleger. Vgl. Poeck/Hacke (1998), S. 732 f. - Die Bezeichnung „Münchhausen-Syndrom“ prägte der Psychiater Sir Richard Asher (1912-1969) nach dem Lügenbaron Münchhausen (The Lancet, 1951). 
Bewusstsein des Begehrens, behindert zu erscheinen oder zu sein, zweitens dem physischen Erscheinungsbild eines Behinderten. ${ }^{34}$

Nach Brunos Modell geht es in all diesen Fällen darum, sich durch eine (vermeintliche) Behinderung Liebe und Anerkennung zu verschaffen, um damit einen Mangel an Liebe in der Kindheit zu kompensieren. Es fällt tatsächlich auf, dass in den Fallbeschreibungen überwiegend kalte, rigide, sexualfeindliche, streng religiöse Elternhäuser dargestellt werden.

\subsection{Psychiatrische Erklärungsmodelle}

\section{Body Dysmorphic Disorder (BDD) und Zwangsstörung}

BIID hat offenbar Gemeinsamkeiten mit Body Dysmorphic Disorder (BDD) bzw. Dysmorphophobia. ${ }^{35}$ Personen mit BDD leiden unter unkontrollierbaren, zwanghaften Gedanken über ihre äußere Erscheinung, mit der nach ihrer Überzeugung etwas nicht stimmt. Angst und Schamgefühle steigern sich vor allem in Situationen, in denen ihr ,Makel ${ }^{*}$ von anderen bewertet werden könnte. Meist ist der leichte oder eingebildete Makel auf der Haut, dem Haar oder der Nase lokalisiert. Bei Männern mit BDD geht es außerdem um die Genitalien, die Körpergröße, die Beine und die Brust, bei Frauen mit BIID um das Gewicht, die Hüften, Beine und Brüste. Die Krankheitseinsicht ist meistens gering; viele beharren mit wahnhafter Intensität darauf, dass sie einen körperlichen Makel hätten. Die zwanghafte gedankliche Beschäftigung mit dem Makel führt meist auch zu einer zwanghaften, zeitraubenden Beschäftigung mit der Inspektion, dem Korrigieren bzw. Verbergen des ,Makels' durch Selbstbespiegelung, Schminke, Kleidung etc. Die Störung ist klinisch mit Zwangsstörungen, Depressionen, Soziophobie, Essstörungen, Substanzmissbrauch, Feindseligkeit und Suizidalität korreliert. Viele BDD-Patienten versuchen, durch kosmetische Operationen ihren ,Makel' zu beseitigen - vor allem mittels Nasenoperationen, Lippenaufspritzung und Brustvergrößerungen. Die meisten sind jedoch unzufrieden mit den Ergebnissen der chirurgischen Korrekturen und erreichen dadurch keine Verbesserung des Selbstbildes und der Lebensqualität. Dagegen kann das Befinden in vielen Fällen mit Serotoninwiederaufnahmehemmern und kognitiver Verhaltenstherapie verbessert werden. Wegen der Nutzlosigkeit chirurgischer Eingriffe bei BDD-Patienten, aber auch weil sich die Fälle häufen, in denen diese Patienten Chirurgen mit Gerichtsprozessen oder sogar körperlich attackieren, setzt sich allmählich der Konsens durch, dass Body Dysmorphic Disorder eine Kontraindikation für kosmetische Chirurgie sein sollte. ${ }^{36}$

Im Gegensatz zu BDD-Patienten glauben BIID-Patienten allerdings meist nicht, dass der Körperteil, auf den sich ihre zwanghaften Gedanken richten,

34 Das erste Kriterium erfüllen Wannabes und erfolgreiche Wannabes, aber nicht Devotees und Personen mit Factitious Disorder. Das zweite Kriterium erfüllen Successful Wannabes und Personen mit Factitious Disorder, aber nicht Wannabes und Devotees. - Vgl. Bruno (1997), S. 258.

35 Zu BDD vgl. Crerand/Franklin/Sarwer (2006); Dyl et al. (2006).

$36 \mathrm{Vgl}$. Crerand/Franklin/Sarwer (2006). 
hässlich oder missgestaltet sei; dies spricht gegen die These, dass der Amputationswunsch auf BDD basiert. ${ }^{37}$

Braam et al. (2006) folgern, dass Apotemnophilie teils eine Zwangsstörung, teils eine Body Dysmorphic Disorder sei, sich aber nicht darauf beschränken lasse: Zusätzlich liege eine Identitätsstörung vor. Sie schlagen ein paradoxes Handicap-Modell vor: Demnach müssen BIID-Patienten mit einem überflüssigen Körperteil fertig werden und haben Nachteile durch ein zeitaufwendiges Bewältigungsverhalten (coping behavior). ${ }^{38}$

\section{Identitätsstörung wie Transsexualität}

Michael First (2004) vertritt die These, dass BIID im Wesentlichen eine Identitätsstörung sei. Dafür spricht, dass fast zwei Drittel der 52 von ihm interviewten BIID-Patienten als Hauptgrund für ihren Amputationswunsch angaben, dass sie damit ihre wahre Identität als Amputierter herstellen wollten. Sie begründen ihren Amputationswunsch mit Aussagen wie, dass sie sich erst nach der Amputation eines bestimmten Körperteils „,vollständig“ fühlen würden, dass sie mit zwei Armen und zwei Beinen „übervollständig“ wären, dass sie sich wie ein Amputierter mit natürlichen Prothesen fühlten und sie diese loswerden wollten, dass sie sich im falschen Körper fühlen, dass ihre „wahre Identität" die eines Amputierten sei.

Für eine Identitätsstörung spricht auch, wie stark sich die Wannabes mit ihrem zwanghaften Wunsch identifizieren; sie sehen den Amputationswunsch meist nicht als seltsam und unerwünscht an, sondern als Teil ihrer Identität, womit sie sich von den meisten anderen Zwangskranken unterscheiden. ${ }^{39}$

Es gibt eine deutliche Korrelation von BIID und Geschlechtsidentitätsstörungen (Gender Identity Disorder, GID)..$^{40}$ Immerhin ein Fünftel der 52 von First untersuchten BIID-Patienten gab an, das Gefühl zu haben, im Körper des falschen Geschlechts zu leben, und mehr als die Hälfte von diesen spielt mit dem Gedanken einer sog. Geschlechtsumwandlung. ${ }^{41}$

First folgert aus seinen Untersuchungen, dass der Wunsch nach Amputation gesunder Körperteile am ehesten der Diagnose Gender Identity Disorder entspricht, mit der er mehrere Merkmale teilt:

1. die Unzufriedenheit mit der anatomischen Identität,

2. ein innerer Sinn der gewünschten Identität,

3. der Beginn in der Kindheit oder frühen Adoleszenz, ${ }^{42}$

37 Vgl. Baynes/Levy (2005), S. 78.

38 Vgl. Braam et al. (2006), S. 37.

$39 \mathrm{Vgl}$. Johnston/Elliot (2002), S. 432.

40 Der Begriff Gender Identity Disorder ersetzt seit den 1990er Jahren den Begriff Transsexualität im Diagnostischen und Statistischen Handbuch Psychischer Störungen (DSM-IV); dagegen werden in der Internationalen Klassifizierung von Krankheiten der Weltgesundheitsorganisation (ICD-10) beide Begriffe synonym verwendet. Der Begriff Transsexualität findet sich dort in der Klasse F (Psychische Störungen und Verhaltensstörungen), F64.0.

41 Vgl. First (2004), S. 7.

42 Von den 52 BIID-Patienten, die First befragt hat, gaben alle bis auf einen (der sich in vielerlei Hinsicht von den übrigen unterschied) an, den Amputationswunsch seit der Kindheit oder frühen Jugend zu haben. - Vgl. First (2004), S. 6. 
4. die manchmal erfolgreiche Therapie durch Amputation,

5. das Imitieren der Wunschidentität (Transvestitismus bzw. Pretending),

6. häufig eine paraphile sexuelle Erregung durch die Vorstellung der Wunschidentität. $^{43}$

Eine weitere Gemeinsamkeit von Mann-zu-Frau-Transsexuellen und BIID-Patienten ist die klischeehafte Vorstellung von ihrem Ideal: So wie jene ein stereotypes Bild von Weiblichkeit haben (schwach, hilflos, besessen vom Aussehen), ${ }^{44}$ so vertreten diese ein stereotypes Bild vom Amputierten (schwach, feminin, aber glücklich); dementsprechend unzufrieden sind beide mit den echten Vertretern ihres Ideals.

So wie GID eine Dysfunktion bei der Entwicklung der Geschlechtsidentität darstellt, ist BIID nach Firsts Modell eine Dysfunktion bei der Entwicklung der Körperidentität. First hat die neue diagnostische Kategorie Body Integrity Identity Disorder vorgeschlagen und fordert, sie in den neuen DSM-Katalog (DSM-V) aufzunehmen. ${ }^{45}$ Dies propagieren auch die Befürworter von WunschAmputationen Robert Smith, Gregg Furth und Annemarie Bridy.$^{46}$ Dabei haben sie die Definition von GID fast 1:1 für die Definition von Body (Integrity) Identity Disorder übernommen - unter Ersetzung von „male“ durch „able-bodied“ und „female“ durch „disabled“. ${ }^{47}$

Auch BIID-Gruppen haben diese Parallele übernommen: Das Internetforum www.transabled.org verwendet den Neologismus transabled analog zum erfolgreichen Vorbild transgender.

\subsection{Neurologische Erklärungsmodelle}

Wenn ein Amputationswunsch aus dem Gefühl der Fremdheit eines bestimmten Körperteils hervorgeht und nicht aus sexuellen Wünschen oder einer psychiatrischen Störung, dann ist eine neurologische Störung zu vermuten, und zwar eine Störung des Körperbildes.

Das Körperbild basiert auf einer Projektion des gesamten Körpers auf bestimmte motorische und somatosensorische Areale (Gyrus praecentralis bzw. Gyrus postcentralis), auf denen sich eindeutige Orts-Funktionszusammenhänge befinden. Die Nachbarschaftsverhältnisse des Körpers sind im Projektionsfeld erhalten; allerdings sind die Größenverhältnisse stark verzerrt. Beispielsweise

43 Vgl. First (2004), S. 8 f.

44 Vgl. Bayne/Levy (2005), S. 81.

45 Vgl. First (2004), S. $8 \mathrm{f}$.

46 Vgl. Bridy (2004), S. 149-151; Furth/Smith (2000), S. 87 f.

47 Furth/Smith (2000), S. 87 f.: „Diagnostic features: There are two components of Body Identity Disorder, both of which must be present to make the diagnosis. There must be evidence of a strong and persistent disability identification, which is the desire to be, or the insistence that one is, internally, disabled (Criterion A). The disability identification must not merely be a desire for any perceived cultural advantages of living with a disability. There must also be evidence of permanent discomfort about living as an able-bodied person, or a sense of inappropriateness in that same role (Criterion B). The diagnosis is not made if the condition is better explained by another medical or psychiatric diagnosis (Criterion C). To make the diagnosis, there must be evidence of clinically significant distress or impairment in social, occupational, or other important areas of functioning (Criterion D)." 
sind die Hände übermäßig vergrößert; der gesamte Rumpf dagegen stark verkleinert. Je genauer die einzelnen Körperteile gesteuert werden müssen, desto ausgedehnter ist ihre Repräsentation im Gehirn. ${ }^{48}$ Dieses Körperabbild wird als Homunkulus (kleiner Mensch) bezeichnet. Reizt man bestimmte Gebiete im Gyrus praecentralis, löst dies Bewegungen der zugehörigen Körperteile aus; reizt man dagegen bestimmte Gebiete im Gyrus postcentralis, werden entsprechende Körperempfindungen hervorgerufen. Das Körperbild ist eine bewusst zugängliche Repräsentation des eigenen Körpers, die aus vielen Quellen resultiert, insbesondere aus der visuellen, propriozeptiven und taktilen Erfahrung; es ist die Basis des Selbstbildes. ${ }^{49}$ Es kann durch periphere oder zentrale Störungen beeinträchtigt werden. ${ }^{50}$

\section{Periphere Störung durch Verletzung oder Ruhigstellung von Gliedmaßen}

Der bekannte britische Neurologe Oliver Sacks hat selbst erlebt, dass ihm eins seiner Beine plötzlich so fremd wurde, dass er zeitweise dessen Amputation wünschte:

„Ich würde an einen Rollstuhl gefesselt und auf erniedrigende Weise von anderen abhängig sein, mit einem Bein, das nutzlos und gleichzeitig so ,fremd' war, so inwendig amputiert, daß es am besten und einfachsten sein würde, es dann auch tatsächlich zu amputieren, weil mich dieser Schritt wenigstens davon erlösen würde, ein völlig nutzloses, funktionsloses, ja tatsächlich totes Glied mit mir herumzuschleppen. Man würde es getrost entfernen können, so wie man ein abgestorbenes Bein entfernte, denn es war ja praktisch wirklich abgestorben - es war neutral, funktional und existentiell tot. ${ }^{\text {(51 }}$

Vorangegangen waren eine schwere Verletzung der Muskeln und Nerven des linken Oberschenkels infolge eines Unfalls, eine Operation unter Vollnarkose sowie das Eingipsen des Beines. Das Besondere dieses Falles ist, dass hier ein Neurologe selbst eine schwere neurologische Störung erlitten hat. Die existentiellen Erfahrungen durch die Erkrankung, die Verzweiflung, die Hilflosigkeit der Medizin sowie die überraschende Genesung haben Sacks zum einen dazu veranlasst, neurologische Störungen des Körperbildes zu erforschen, zum anderen, seine persönlichen Erfahrungen in einem Buch darzustellen, ${ }^{52}$ das sogar zu einem Bestseller wurde.

Sacks glaubte zunächst, er habe während der Operation einen Gehirnschlag im rechten Scheitellappen erlitten, wodurch sein linkes Bein aus seinem Körperbild „gelöscht“ worden sei. ${ }^{53}$ Nach seiner Genesung korrespondierte er mit dem bekannten russischen Neurologen und Neuropsychologen Alexander Lurija. Dieser vermutete, dass die Störung des Körperbildes allein durch die Ver-

48 Vgl. Zimbardo (1995), S. 140-142.

$49 \mathrm{Vgl.}$ Baynes/Levy (2005), S. 76.

50 Vgl. Sacks (2004), S. 205-222.

51 Vgl. Sacks (2004), S. 80.

52 Vgl. Sacks: A leg to stand on (1984); dt. Übersetzung: Der Tag, an dem mein Bein fortging (1989).

53 Vgl. Sacks (2004), S. 77. 
letzung der Muskeln und der peripheren Nerven sowie die anschließende Ruhigstellung hervorgerufen worden sei.

„Der Körper ist eine Einheit von Handlungen, und wenn ein Teil des Körpers von der Handlung abgespalten ist, so entwickelt er sich zu etwas, Fremdem“ und wird nicht mehr als Teil des Körpers empfunden. ${ }^{454}$

Weil der Organismus ein einheitliches System sei, könne ein Zusammenbruch des gesamten Systems erfolgen, ganz gleich, ob die ursprüngliche Störung zentraler oder peripherer Natur sei. Sowohl Störungen in dem Gehirnareal, das das Bild des betreffenden Körperteils generiert, als auch Störungen in dessen peripheren Nerven bzw. im Rückenmark könnten Derealisierung, Gleichgültigkeit und Unaufmerksamkeit gegenüber dem betreffenden Körperteil hervorrufen. Ärzte stehen dieser Störung häufig abweisend gegenüber, zumal die meisten Patienten sie nicht richtig beschreiben könnten..$^{55}$ Sacks vermutet im Anschluss an Babinski, dass das Fremdwerden von Körperteilen infolge einer peripheren Verletzung weder hysterisch noch im neuroanatomischen Sinne organisch sei, sondern eine tiefgehende, posttraumatische physiologische Störung darstellt: Es beruhe auf einem „Schock“ (einer Reflexwirkung wahrscheinlich synaptischer Art) und einer sich verstärkenden Hemmung spinaler und peripherer Mechanismen. Zusätzlich sei aber noch eine Störung auf einer höheren Ebene, ähnlich einer Anosognosie, zu vermuten. ${ }^{.6}$ Schon Leontjew und Zaporožec haben während des Zweiten Weltkriegs ein Syndrom beschrieben, das sie bei 200 Soldaten mit verletzten und operativ wiederhergestellten Händen festgestellt hatten: Diese fühlten sich fremd an, wie nachgemachte Hände, die an den Handgelenken befestigt waren. Leontjew und Zaporožec sprachen von einer ,inneren Amputation“ durch eine „Dissoziation der gnostischen Systeme“, die die Hände normalerweise steuern, infolge der Ruhigstellung. ${ }^{57}$

Sacks untersuchte Hunderte von Patienten, bei denen infolge eines Unfalls oder einer Ruhigstellung einer Cliedmaße diese dem Patienten so fremd geworden war, dass er sie nicht mehr spürte bzw. nicht mehr als seine eigene empfand. Bei einer Patientin, deren Bein nach einer Hüftfraktur und einer langen Ruhigstellung „fort“ war, stellte er fest, dass die Muskeln dieses Beins völlig atonisch waren und keinerlei elektrische Impulse darin nachweisbar waren. Es waren auch keine evozierten Potentiale der sensorischen Hirnrinde messbar: „ein objektiver Riß im Körper-Bild““. ${ }^{8}$ Auch bei ca. 5o Patienten mit schweren peripheren Neuropathien, vor allem durch Diabetes verursacht, fand Sacks dasselbe Bild: gravierende sensorische und manchmal motorische Behinderungen der Hände und Füße sowie das Gefühl, dass diese fehlten oder fremde, an Arm- bzw. Beinstümpfen festgemachte Objekte seien. Untersuchungen der evozierten Potentiale zeigten schwere Beeinträchtigungen oder

\footnotetext{
54 Zitat aus einem Brief von Lurija an Sacks in Sacks (2004), S. 205.

55 Vgl. Sacks (2004), S. 206.

56 Vgl. Sacks (2004), S. 208 und 231.

57 Vgl. Sacks (2004), S. 215.

58 Vgl. Sacks (2004), S. 209.
} 
das Fehlen von perzeptueller Information und Repräsentation in den entsprechenden Bereichen der sensorischen Hirnrinde. Auch ca. 200 Patienten mit Rückenmarksverletzungen oder -erkrankungen berichteten Sacks von Gefühlen der Fremdheit, des Fehlens oder plötzlichen Verschwindens bestimmter Körperteile.$^{59}$ In manchen Fällen führen periphere Nervenschmerzen zum Wunsch nach Amputation. ${ }^{60}$

Das Fremdwerden von Gliedmaßen ist gewissermaßen komplementär zu Phantomgliedern. Im ersten Fall ist die Gliedmaße vorhanden, aber das Gefühl für sie fehlt, im zweiten Fall ist das Gefühl für die Gliedmaße da, aber diese fehlt. Oliver Sacks wurde durch einen Mitpatienten, dem wegen Diabetes ein Bein amputiert worden war, auf die Idee gebracht, die beiden Phänomene zu kombinieren, um Phantomschmerzen nach Amputationen zu verhindern:

\begin{abstract}
„,Dieses verdammte Phantom', sagte er. ,[...] Gibt es denn keine Möglichkeit, das zu vermeiden? Herrgott ja', rief er, ,Sie sind die Lösung. Bevor sie es abgeschnitten haben, hätten sie mir bloß eine Narkose verpassen, die Nerven durchtrennen und das Bein in einen Gips packen müssen. Dann hätte ich das Gefühl dafür verloren, so wie Sie. Und dann, wenn das Gefühl verschwunden gewesen wäre, dann hätte man es abschneiden können!' [...] Die Idee erschien mir gut, ja glänzend. Mir kam der Gedanke, ich könnte sie ,medizinisch formulieren' und unter seinem Namen an die Fachzeitschrift, Lancet' schicken: ,Einfache Prophylaxe gegen die Entstehung von Phantomen. “'61
\end{abstract}

Sacks hat festgestellt, dass schwere Störungen des Körperbildes infolge peripherer Verletzungen, Erkrankungen oder Störungen recht verbreitet und fast unvermeidbar sind, wenn eine hinreichende Unterbrechung des peripheren Empfindungs- oder Handlungsvermögens vorliegt. ${ }^{62}$

„Jeder Patient mit einer schweren Störung des Körper-Bildes litt unter einer ebenso schweren Störung des Körper-Ichs. Es wurde immer deutlicher, daß ein solcher Patient in den betroffenen Gliedmaßen einer tiefgreifenden, mit dem Gefühl der Auflösung oder Zerstörung oder Auslöschung des Seins verbundenen Erfahrung unterworfen ist. Dies geht einher mit einer elementaren Derealisierung und Entfremdung sowie gleichermaßen elementaren Gefühlen von Angst und Entsetzen. Wenn sie das Glück haben zu genesen, wird dies von einem ebenso elementaren Gefühl der ,Re-realisation‘ und Freude gefolgt. Eine jede solche Erfahrung

59 Vgl. Sacks (2004), S. $209 \mathrm{f}$.

60 Die Medizinethikerin Denise Dudzinski (2005) beschreibt eine 50-jährige Patientin, die 10 Jahre nach einer Verletzung ihres Handgelenks unter unerträglichen chronischen Schmerzen im betreffenden Arm litt (complex regional pain syndrom, CRPS), die sie daran hinderten, sich umzukleiden und mit ihrem Enkel zu spielen. Sie wollte wegen dieser Beeinträchtigungen, dass ihr Arm amputiert werde, obwohl die behandelnden Ärzte der Ansicht waren, dass die chronischen Schmerzen nicht verschwinden, sondern in Phantomschmerzen übergehen würden. Obwohl sie selbst von der Amputation keine Schmerzlinderung erhoffte, beharrte sie darauf, weil der Arm ihr so im Weg war, dass sie sich dadurch im sozialen Leben stark beeinträchtigt fühlte. Andere Verfahren zur Schmerzreduktion lehnte sie ab bzw. kamen aus medizinischer Sicht nicht in Frage. Nachdem mehrere Chirurgen die Amputation abgelehnt hatten, amputierte schließlich ein Chirurg ihren Arm. Sie ist weiterhin in schmerztherapeutischer Behandlung.

61 Vgl. Sacks (2004), S. $175 \mathrm{f}$.

62 Vgl. Sacks (2004), S. 211. 
ist [...] eine elementare Veränderung der Identität oder ,Selbstheit' auf einer ganz klar umrissenen, organischen neurologischen Grundlage. ${ }^{\text {“63 }}$

Die Immobilisierung oder orthopädische Ruhigstellung von Gliedmaßen birgt die Gefahr, dass komplexe Bewegungen, die nicht ausgeführt werden können, innerhalb weniger Wochen ,vergessen“ und auch neurologisch unmöglich werden. ${ }^{64}$ Das Körperbild ist nicht statisch und ,fest verdrahtet', sondern verändert sich je nach Mobilität, Beanspruchung und Erfahrung der entsprechenden Körperteile kontinuierlich; es kann sich sogar innerhalb weniger Stunden an veränderte Gegebenheiten anpassen. Nach einer Amputation, einer Lähmung oder Deafferentierung wird ein Teil des Körperbildes ,ausradiert und dessen Platz von benachbarten Teilen eingenommen. ${ }^{65}$ Merzenich et al. (1984) haben gezeigt, dass nach der Amputation eines Körperteils das diesem zugeordnete somatosensorische Cortexareal von benachbarten Körperteilen übernommen wird.

„Die Experimente [von Merzenich] zeigen, daß es für keinen Körperteil einen festgelegten, ,reservierten' Bereich gibt, also auch keine bestimmte ,Hand'-Area. Wenn eine Hand für eine gewisse Zeit deafferentiert oder inaktiviert ist, verliert sie ihren Platz im sensorischen Rindenfeld. Dieser wird innerhalb von Stunden oder Tagen überlagert von der Repräsentation der anderen Körperteile, so daß im Kortex nun eine neue ,handlose' Abbildung des Körpers existiert." ${ }^{66}$

Eine lokal begrenzte, periphere Störung kann also zu einer massiven Störung des Bewusstseins führen, denn die höheren Ebenen des Bewusstseins basieren auf den basaleren Ebenen. Die Entfremdung von Gliedmaßen ist ein Beispiel für ein solches neuropsychologisches Syndrom. Neuropsychologische Syndrome richten sich von unten nach oben (von der neuronalen Ebene zur Bewusstseinsebene) im Gegensatz zu neurotischen oder hysterischen Störungen, die von oben nach unten gerichtet sind. ${ }^{67}$

Sacks konnte zahlreichen Patienten mit Bewegungstherapie, insbesondere mit Musiktherapie helfen. Die Reintegration der abgespaltenen sensorischen Systeme ist nur durch den Gebrauch der betroffenen Körperteile möglich. Doch da die Patienten diese meist nicht bewusst steuern können, müssen sie überlistet werden, damit sie die Bewegungen spontan initiieren. Ein idealer Auslöser für komplexe, automatisierte Bewegungen ist Musik: Die „fremden“ Körperteile fügen sich beim Hören von Musik häufig spontan in einen komplexen Bewegungsablauf ein. Sacks selbst wurde dadurch geholfen, dass ein Bademeister ihn ins Wasser stieß und zu einem Wettschwimmen animierte. Seitdem konnte er das Bein wieder normal benutzen. Diese Methode funktioniert auch

63 Vgl. Sacks (2004), S. 211.

64 Heute wird dieser Tatsache in der Behandlung von verletzten Gliedmaßen dadurch Rechnung getragen, dass sie möglichst kurzzeitig ruhig gestellt werden und so bald wie möglich mit Bewegungstherapie begonnen wird. Vgl. Sacks (2004), S. 233.

65 Vgl. Sacks (2004), S. 226-228 und 234-236; Merzenich et al. (1984).

66 Vgl. Sacks (2004), S. 234.

67 Vgl. Sacks (2004), S. 243. 
bei Hunden, die infolge einer längeren Ruhigstellung eines Beines nicht mehr richtig laufen können. ${ }^{68}$

Auffallend viele BIID-Patienten berichten von Verletzungen mit nachfolgender Ruhigstellung bzw. über die zeitweilige Lähmung derjenigen Cliedmaßen, deren Amputation sie später wünschten. ${ }^{69}$ Möglicherweise war der zeitweilige Nichtgebrauch der Cliedmaßen der Ursprung des Amputationswunsches. Auch bei den Patienten, bei denen am Anfang der Claube stand, dass ihre Sehnsucht nach Liebe und Beachtung durch eine Behinderung erfüllt würde, könnte das Deaktivieren von Cliedmaßen zu BIID geführt haben: Das regelmäßige Ruhigstellen oder Abbinden von Gliedmaßen zum Zweck des Pretending kann periphere Störungen wie Neuropathien hervorrufen und schließlich eine manifeste Störung des Körperbildes mit Auslöschung des betreffenden Körperteils bewirken. Dann ist der Wunsch nach dessen Amputation die notwendige Folge.

\section{Pötzl-Syndrom}

Ein Gehirnschlag oder ein Gehirntumor im rechten Scheitellappen kann bewirken, dass die linke Körperhälfte oder Teile davon ignoriert werden bzw. als fremd, unwirklich oder sogar als Teil des Körpers einer anderen Person empfunden werden. Dieses Phänomen ist als Pötzl-Syndrom bekannt. ${ }^{70}$

Otto Pötzl hat groteske Beispiele beschrieben, z. B. von einem Patienten, der die Krankenschwester bat, mit dem Frühstückstablett auch das Bein mitzunehmen, sowie von einem Patienten, der während einer Bahnfahrt seinen Nachbarn aufforderte, dessen Hand von seinem Bein zu nehmen, und dabei auf seine eigene Hand deutete. Während die Affekte und Reaktionen dieser

68 Vgl. Sacks (2004), S. 197-199, 215f. - Das Phänomen der Auslöschung von Gliedmaßen aus dem Körperbild ist auch Tierärzten bekannt. Kühe, die während der Entbindung eine Spinalanästhesie erhalten, beachten ihren Hinterleib nicht mehr und nehmen von der Geburt ihres Kalbes keine Notiz. Anders als Tiere können Menschen aber ihre Aufmerksamkeit auf den gelöschten Körperteil richten; erst dies führt zu dem Gefühl der Fremdheit der entsprechenden Körperteile. Vgl. auch S. 237-243, bes. S. 243: „Entfremdung ist ein blinder Fleck im primären Bewusstsein, der vom Bewusstsein höherer Ordnung wahrgenommen wird."

69 Bensler et al. (2003): Der Patient mit dem Wunsch nach Beinamputation hatte zahlreiche (zumindest teilweise selbst verursachte) Verletzungen der unteren Extremitäten.

Bruno (1997), Fall 2: Die Patientin klagte über Arm- und Beinschwäche sowie Gleichgewichtsstörungen. Bruno diagnostizierte Factitious Disability Disorder. Im Alter von einem Jahr hatte sie Kinderlähmung. Als Kind fiel sie häufig hin. Im Alter von 21 Jahren hatte sie eine Hüftoperation, um das häufige Hinfallen zu beheben. Mit Anfang 40 erhielt sie verschiedene orthopädische Stützen für Kniee und Fußgelenke wegen deren Instabilität und Schmerzen.

Dudzinski (2005): Die Patientin hatte eine Verletzung des Handgelenks, die zu einem chronischen Schmerzsyndrom führte; weil sie den betreffenden Arm seitdem als völlig nutzlos und störend empfand, wünschte sie dessen Amputation.

Money/Jobaris/Furth (1977), Fall 1: Der Patient, der heimlich transsexuell sowie bisexuell und Amputationsfetischist war, hatte im Alter von 2 Jahren eine schwere Verbrühung seines linken Beins erlitten und konnte danach ein Jahr nicht laufen. Er wünschte die Amputation seines linken Beins.

Money/Jobaris/Furth (1977), Fall 2: Der Patient war mit einer leichten Fehlbildung des rechten Fußes zur Welt gekommen, durch die er einen fehlerhaften Gang entwickelte, für den er von seinem Vater streng kritisiert wurde. In der Pubertät wurde die Fehlbildung chirurgisch korrigiert, und der Gang normalisierte sich. Seit dem Alter von 11 Jahren wünschte der Patient die Amputation des rechten Fußes. Im Alter von 20 Jahren erlitt er bei einem Arbeitsunfall einen Bruch des rechten Beins. Die Behinderung durch den Gips und das Gehen mit Krücken genoss er sehr.

70 Vgl. Sacks (2004), S. 76-80. 
Patienten in allen anderen Bereichen normal erscheinen, zeigen sie gegenüber ihren betroffenen Gliedmaßen eine außergewöhnliche Gleichgültigkeit. ${ }^{71}$

Auch Oliver Sacks beschreibt einen solchen Fall: Ein junger Mann war stationär aufgenommen worden, weil sein linkes Bein sich auf einmal „träge“ anfühlte. Am nächsten Tag stellte er beim Erwachen entsetzt fest, dass ein fremdes Bein in seinem Bett lag, offenbar das Bein einer Leiche. Weil gerade Silvester war, nahm er an, eine Krankenschwester habe sich einen makabren Scherz erlaubt. Er versuchte, das Bein aus dem Bett zu werfen, und stürzte dabei selbst hinaus; nun schien das Bein an ihm festgewachsen zu sein. Er sagte, das Bein sehe „absolut grauenhaft“ aus; es sei eine „Fälschung“ seines eigenen Beins; dieses sei „verschwunden“, habe „sich in Luft aufgelöst“. Es wurde ein großer Gefäßtumor über dem rechten Scheitellappen diagnostiziert, der in der Nacht zu bluten begonnen und das „Beinzentrum“ gelöscht hatte. Dem Patienten wurde die „Auslöschung“ seines Beines aus dem Körperbild erklärt und gesagt, dass es nach der Entfernung des Tumors wahrscheinlich „zurückkehren“" werde. Der Patient konnte sich aber weder vorstellen, dass das Bein zurückkehren würde, noch daran erinnern, dass er es jemals gehabt habe. Das Bein war, so urteilt Sacks, nicht nur verloren gegangen, sondern hatte sogar seinen angestammten Platz verloren. Trotzdem „kehrte“ es nach der Gehirnoperation wieder „zurück““ ${ }^{72}$ Sacks konstatiert:

„Und in der Tat lag bei innen ja ein außerordentlich bemerkenswerter ,Zerfall“ vor - eine Dissoziation, die nicht nur neuraler, sondern auch emotionaler und ,existentieller' Natur war. Dies war jedoch nicht die Folge einer ,Verdrängung' eines Gedankens und Gefühls, sondern das Resultat einer neuronalen Unterbrechung. “73

\section{Neurologische Störung wie das Fremde-Hand-Syndrom}

Auch das Fremde-Hand-Syndrom ${ }^{74}$ ähnelt den Symptomen von BIID. Dieses Syndrom ist eine sehr seltene neurologische Störung, von der erst ca. 50 Fälle dokumentiert wurden. Personen mit dem Fremde-Hand-Syndrom empfinden eine Hand als nicht zu ihnen gehörig; im Gegensatz zur BIID entzieht sich beim Fremde-Hand-Syndrom die betreffende Hand vollständig der willentlichen Kontrolle des Patienten und bewegt sich scheinbar autonom. ${ }^{75}$ Häufig

71 Vgl. Sacks (2004), S. 76-78.

72 Vgl. Sacks (2004), S. 73-79.

73 Vgl. Sacks (2004), S. 78.

74 Vgl. hierzu Biran/Chatterjee (2004), Ovadia (2006), Pappalardo et al. (2004), Scepkowski/Cronin-Golomb (2003). Andere Bezeichnungen dieses Syndroms sind Alien-Hand-Syndrom, la main étrangère und Dr. Strangelove-Syndrom.

75 Ovadia (2006), S. 64 f., zitiert einen Patienten, der durch einen Schlaganfall eine Schädigung im rechten prämotorischen Areal und im vorderen Teil des Corpus callosum erlitten hatte: „Seit ich wieder zu Hause bin, ist diese Hand wie besessen. Ich kann sie überhaupt nicht kontrollieren, sie macht einfach, was sie will! [...] Es ist ein wahrer Albtraum. Wenn ich mit der rechten Hand ein Stück Fleisch auf die Gabel spieße, pflückt es mir die linke wieder von den Zinken. Wenn das Telefon klingelt und ich die rechte Hand ausstrecke, um den Hörer abzunehmen, schießt die linke hervor und hält das Telefon einfach fest. Ich schaffe es nicht, den Hörer ans Ohr zu führen. Das Gleiche beim Schach - ich bin ein leidenschaftlicher Spieler: Ständig führt die linke Hand absurde Züge aus, die ich mit rechts korrigieren muss. Meine Freunde haben sich daran gewöhnt und nehmen es hin. Doch gegen den Computer spiele ich schon nicht mehr, weil ich die Züge da nicht rückgängig machen kann. Es ist, als ob sich meine Hände streiten: Die rechte folgt meinem Willen, die linke verhält sich wie ein aufsässiges Kind. [...] Manchmal bekomme ich eine Ohrfeige - von meiner eigenen Hand! [...] Dabei fühlte ich mich wie in einem Horrorfilm, eine teuflische Macht schien von mir Besitz ergriffen zu haben. Nur dass sie sich in meinem Fall mit einem Arm zufrieden gab." 
muss der Patient seine ,fremde Hand" mit physischer Gewalt kontrollieren, z. B. indem er sie am Körper festbindet. Die Patienten zeigen häufig einen Autokritizismus bezüglich des betroffenen Körperteils. ${ }^{76}$

Kurt Goldstein (1878-1965) hat das Fremde-Hand-Syndrom als Erster 1908 bei einer Patientin beschrieben, deren Hand nach einem Schlaganfall einen Eigenwillen entwickelt zu haben schien. Einmal krallte die Hand sich so fest um den Hals der Frau, dass zwei Männer sie losreißen mussten, um die Frau vor dem Ersticken zu retten. Sie selbst glaubte, ein Dämon sei in sie gefahren. In der Obduktion der Patientin entdeckte Goldstein eine Läsion der rechten Hemisphäre und des Corpus callosum. Ähnliche Symptome traten bei einigen $\mathrm{Pa}$ tienten auf, denen wegen Epilepsie der Corpuscallosum durchtrennt worden war, oder die dort oder im medialen frontalen Cortex einen Tumor oder eine Blutung aufgrund eines geplatzten Aneurysmas oder einer Verletzung hatten.

Klinisch lassen sich vier Kategorien des Syndroms unterscheiden, die durch die oben beschriebenen klinischen Störungen bzw. eine Kombination daraus hervorgerufen werden können: ${ }^{77}$

1. Intermanueller Konflikt: Die nichtdominante Hand widersetzt sich den Aktionen der dominanten Hand. ${ }^{78}$

2. Fremde Hand: Der Patient empfindet eine Hand als fremd, nicht zugehörig und bedeutungslos. Wenn er sie nicht sieht, kann er sie nicht als seine eigene Hand identifizieren.

3. Anarchische Hand: Eine Hand agiert gegen die bewussten Intentionen des Patienten.

4. Überflüssige Hand: Der Patient hat das Gefühl einer zusätzlichen Hand.

Je nach Läsionsort werden drei Subtypen des Fremde-Hand-Syndroms unterschieden:

1. Callosaler Subyp: Bei einer Schädigung des Corpus callosum ${ }^{79}$ tritt meist ein intermanueller Konflikt auf, d. h. die linke (nichtdominante) Hand widersetzt sich der rechten (dominanten) Hand. ${ }^{80}$ Die Ursache dafür ist vermutlich, dass i.d. R. die linke (dominante) Gehirnhälfte auch die komplexen feinmotorischen Bewegungen insgesamt koordiniert. Ist das Corpus callosum defekt, wird die linke Hand nur noch von der rechten Gehirnhälfte gesteuert und verliert die Steuerung durch die linke, die Bewegungen koordinierende Gehirnhälfte. Das führt dazu, dass die linke Hand der bewussten Kontrolle entzogen wird und sich sogar den Bewegungen der rechten Hand widersetzt. Typischerweise gibt es dabei zahlreiche weitere Anzeichen einer unterbrochenen Verbindung der beiden Hirnhemisphären.

76 Vgl. Pappalardo et al. (2004), S. 177; Biran/Chatterjee (2004), S. 292.

77 Vgl. Pappalardo et al. (2004), S. 179f.; Biran/Chatterjee (2004).

78 Beispielsweise knöpft die linke Hand ein Hemd wieder auf, das die rechte gerade zuknöpft, oder sie schlägt eine Zeitung zu, die die rechte soeben aufgeschlagen hat. Vgl. Biran/Chatterjee (2004), S. 293 f.

79 Corpus callosum = Balken, der die linke und die rechte Gehirnhälfte miteinander verbindet.

80 Scepkowski/Cronin-Golomb (2003) betonen, dass alle bisher beschriebenen Fälle des Fremde-Hand-Syndroms bei Rechtshändern oder beidhändigen Personen aufgetreten sind (S. 262 f.). Schon 1974 hatte Lurija darüber spekuliert, ob Entfremdungen nur bei linksseitigen Gliedmaßen auftreten können. Vgl. Sacks (2004), S. $226 \mathrm{f}$. 
2. Frontaler Subtyp: Wenn das Corpus callosum intakt ist, aber bestimmte Teile des Frontallappens geschädigt sind, die Bewegungen planen und ausführen, insbesondere die prämotorischen und die supplementär-motorischen Areale (SMA), ist die rechte Hand betroffen; sie zeigt unkontrollierbare Greifreflexe und zwanghafte Manipulationen an Gegenständen. Ein intermanueller Konflikt ist dabei selten. ,Fremde‘ Bewegungen werden häufig durch visuelle oder taktile Stimulation ausgelöst. Auch eine magnetische Apraxie kann auftreten, bei der die linke Hand von der rechten scheinbar angezogen wird. Die Ursache dieser Symptome liegt wahrscheinlich darin, dass durch die Schädigung eines oder beider SMA (Regionen, die bewusste Bewegungsabläufe steuern) die Hemmung auf das laterale, reaktive prämotorische System ausfällt, so dass dieses unwillkürliche Bewegungen auslösen kann.

3. Posteriorer Subtyp: Eine Läsion in einem Cortexgebiet, das von der rechten posterioren Cerebralarterie versorgt wird, kann zu einem Fremde-HandSyndrom führen, das vor allem durch das Gefühl eines ,wandernden“ oder schwebenden Arms sowie durch unbeabsichtigte Bewegungen gekennzeichnet ist.

Kombinationen dieser Subtypen sind häufig, vor allem nach Schlaganfällen oder Gehirntraumata. Auch bei der Alzheimer-Krankheit wurde dieses Syndrom beobachtet. Meist verschwindet das Fremde-Hand-Syndrom nach wenigen Wochen oder Monaten; in einigen Fällen hält es jahrelang an. Neuropsychologische Rehabilitationsmaßnahmen können die Heilung unterstützen.

\section{Angeborener Mismatch von physischem Körper und Körperbild}

Der Amputationswunsch könnte auch daher rühren, dass der physische Körper und der phänomenale Körper nicht übereinstimmen; dass also eine Diskrepanz (mismatch) besteht zwischen dem Körper, wie er objektiv ist, und dem Körper, wie er subjektiv wahrgenommen wird. Eine solche Diskrepanz besteht bei Asomatognosie. Diese Störung ist durch das Leugnen der Zugehörigkeit einer Extremität gekennzeichnet und kann im Rahmen eines Neglects durch einen Schlaganfall, im Kontext einer Depersonalisation oder durch den Verlust der Propriozeption (Eigenwahrnehmung) auftreten. ${ }^{81}$

Der Chirurg Robert Smith und Karen Fisher nehmen an, dass BIID ein Spiegelbild des Phänomens der Phantomglieder sei und dass dabei der physische Körper und der phänomenale Körper nicht übereinstimmten. Bei BIID habe sich möglicherweise ein körperliches Glied entwickelt, ohne dass das sensorische Bewusstsein dafür entwickelt worden sei.$^{82}$ Auch die Medizinethiker Baynes und Levy (2005) nehmen an, dass bei BIID eine Asomatognosie, eine nichtwahnhafte somatische Entfremdung (non-delusional somatic alienation) vorliege. Dann sei der betroffene Körperteil oder zumindest dessen neuronale Repräsen-

81 Eine neurologische Störung als Ursache von BIID hält auch First (2004) für möglich; er weist auf die Ähnlichkeit mit einem Neglect nach einem Schlaganfall hin (S. 9).

$82 \mathrm{Vgl}$. Fisher/Smith (2000). 
tation tatsächlich nicht gesund.$^{83}$ Damit rechtfertigen sie wie Smith die von diesem vorgenommenen Amputationen (scheinbar) gesunder Gliedmaßen.

Anders als bei Fremde-Hand-Syndrom-Patienten besteht die Störung bei BIID-Patienten seit ihrer Kindheit, möglicherweise aufgrund einer angeborenen Missbildung im Gehirn (z. B. einer Gefäßanomalie) oder der unvollständigen Entwicklung bestimmter Nervenbahnen. Zur Überprüfung dieser These sind Untersuchungen mit funktioneller Bildgebung erforderlich; dies wurde von Ramachandran vorgeschlagen, aber bisher noch nicht realisiert. ${ }^{84}$ Wenn BIID auf einer angeborenen oder früh entwickelten hirnorganischen Störung des Körperbildes basiert, haben BIID-Patienten im Gegensatz zu SchlaganfallPatienten mit Fremde-Hand-Syndrom keine Erinnerung an ein Leben, in dem der betreffende Körperteil in das Körperbild integriert war; sie empfinden daher keinen Verlust, sondern einen störenden Ballast. Dieser Unterschied kann erklären, warum ein und dasselbe Syndrom von den Schlaganfall-Patienten als Störung empfunden, aber von BIID-Patienten als Teil der Identität betrachtet wird. Dieselbe Diskrepanz besteht bekanntlich zwischen angeborener und erworbener Gehörlosigkeit. Könnte den Patienten mit Hilfe bildgebender Verfahren gezeigt werden, was die hirnorganische Ursache ihres Gefühls von Fremdheit einer Gliedmaße ist, könnte allein diese Einsicht schon eine gewisse Erleichterung und Entlastung bringen.

\section{Dysfunktion des rechten Scheitellappens wie bei Somatoparaphrenia}

Nach einer kürzlich publizierten Hypothese von Vilayanur Ramachandran und Paul McGeoch (2007) ist die Ursache von BIID eine Dysfunktion des rechten Scheitellappens. Dies schließen sie aus der großen Ähnlichkeit von BIID mit Somatoparaphrenie, einer Störung infolge eines Schlaganfalls im rechten Scheitellappen (meist bei Patienten mit linkshemisphärischem Sprachzentrum), bei der der Patient überzeugt ist, eine bestimmte eigene Extremität (meist der linke Arm) gehöre einer anderen Person. ${ }^{85}$ Für diese These sprechen auch das Überwiegen linksseitiger Amputationswünsche bei BIID-Patienten, die emotionale Abstoßung des betreffenden Körperteils sowie die Spezifität der gewünschten Amputation (ganz bestimmter Körperteil und ganz bestimmtes Niveau). ${ }^{86}$ Ram-

$83 \mathrm{Vgl.} \mathrm{Baynes/Levy} \mathrm{(2005),} \mathrm{S.} 76 \mathrm{f}$.

84 Auch bei Patienten mit dem Fremde-Hand-Syndrom gab es (bis 2003) noch keine fMRT-Untersuchungen und nur wenige PET-Untersuchungen. - Vgl. Scepkowski/Cronin-Golomb (2003), S. 275.

85 Unter Anosognosie (griech. an = nicht; nosos = Krankheit; gnosis = Erkenntnis) versteht man ein krankhaftes Nichterkennen einer Halbseitenlähmung, einer kortikalen Blindheit, einer Hemianopsie oder Taubheit infolge einer Schädigung bestimmter Gehirnareale, meist in Folge eines Schlaganfalls. Es gibt drei Unterarten der Anosognosie: 1. Asomatognosie (Leugnen der Zugehörigkeit einer Extremität), 2. Somatoparaphrenie (Überzeugung, eine eigene Extremität gehöre einer anderen Person), 3. Anosodiaphorie (Bewertung einer schweren Krankheit als Lappalie). Spricht man den Patienten auf die Störung an, erfolgen Konfabulationen, Entschuldigungen und Rationalisierungen. Die Störung tritt meist bei Patienten auf, die eine rechtshemisphärische Störung haben und deren Sprachzentrum linkshemisphärisch lokalisiert ist. Da Patienten mit Anosognosie von der rechten Hemisphäre keine ihrem bisherigen Weltbild entsprechenden Informationen erhalten, hält die linke Hemisphäre am bisherigen Körperschema fest, und die Störungen werden wegerklärt. - Vgl. http://de.wikipedia.org/wiki/Anosognosie [08.02.2007] und Poeck/Hacke (1998), S. 172.

86 Vgl. auch First (2004), S. 5: Von den 52 interviewten BIID-Patienten hatten $90 \%$ einen ganz spezifischen Amputationswunsch (überwiegend eine Beinamputation oberhalb des Knies). Die Mehrheit (55\%) wollte eine linksseitige Amputation, $28 \%$ eine rechtsseitige, $18 \%$ eine beidseitige Amputation. 
achandran und McGeoch nehmen an, dass bei dieser Störung eine Entkopplung des eigenen Körperbildes, das im rechten Scheitellappen generiert wird, von der physischen Realität des eigenen Körpers auftritt. Sie schlagen einen Test für ihre Hypothese vor: Mit einer Vestibularstimulation mittels einer Kaltwasserspülung lasse sich Somatoparaphrenie für eine Weile beheben ${ }^{87}$ - möglicherweise auch BIID. Die Hypothese könne außerdem mit Hilfe funktioneller Bildgebung und Hautleitfähigkeitsmessungen überprüft werden. Falls die Hypothese zutrifft, könnte BIID mittels thermischer Stimulation des Vestibularsystems kurzzeitig therapiert werden. ${ }^{88}$

\subsection{Multikausale Erklärung und Therapieoptionen}

Wahrscheinlich kann der Wunsch nach der Amputation gesunder Gliedmaßen aus verschiedenen Ursachen resultieren, und bei manchen Patienten könnte eine Kombination von zwei oder mehr Ursachen vorliegen, die sich gegenseitig verstärken. ${ }^{89}$ Daher muss beim Vorliegen eines medizinisch unsinnigen Amputationswunsches eine genaue Differentialdiagnostik erfolgen, da je nach Diagnose unterschiedliche Therapien indiziert sind. Dazu gehören eine genaue Anamnese, eine psychiatrische und eine neurologische Untersuchung sowie ggf. eine Untersuchung mit funktioneller Bildgebung (fMRT oder PET).

Als Ursachen medizinisch unsinniger Amputationswünsche kommen also nach bisherigem Forschungsstand folgende Krankheitsbilder in Frage: 1. eine neurotische Störung, 2. Paraphilie, 3. eine Kombination aus Body Dysmorphic Disorder und Zwangsstörung, 4. eine hirnorganisch bedingte Störung des Körperbildes. Im Folgenden stelle ich die verschiedenen möglichen Ursachen dieser Störung mit den ggf. geeigneten Therapien zusammen:

1. Neurotische Störung, z.B. Factitiuos Disorder oder abgelehnte männliche Homosexualität:: ${ }^{90}$ Der Wunsch, körperbehindert zu sein bzw. zu erscheinen, kann aus einer neurotischen Störung resultieren: zum einen aus einem extremen Wunsch nach Aufmerksamkeit und Liebe, verbunden mit der (irrigen) Annahme, dass körperbehinderte Menschen besonders viel davon bekämen, und mit einem starken Bedürfnis nach Regression (insbesondere nach Rückzug aus dem Berufsleben), ${ }^{91}$ zum anderen aus der Ablehnung der eigenen Homosexualität. ${ }^{92}$ Bei ,nur neurotisch gestörten Apotemnophilen könnte eine Kombination aus Psychotherapie

87 Vgl. auch Ramachandran/Blakeslee (2002), S. 238-247.

88 Vgl. Ramachandran/McGeoch (2007).

89 Diese These vertreten auch Baynes/Levy (2005), S. 77.

$90 \mathrm{Vgl.} \mathrm{Bruno} \mathrm{(1997).}$

91 Beispiele für den Wunsch nach Aufmerksamkeit und Liebe als Ursache für Apotemnophilie sind der Fall 1 von Bruno (1997) sowie die Fälle von Everaerd (1983) und Berger et al. (2005). Beispiele für Regressionswünsche sind der Fall 2 von Bruno (1997) sowie der von Berger et al. (2005) beschriebene Patient, der sich im Rollstuhl als „authentischer und femininer" empfindet (S. 382).

92 Bei den beiden von Money (1977) beschriebenen homosexuellen Autoapotemnophilen liegt offenbar ein Minderwertigkeitskomplex wegen ihrer Homosexualität vor. Sie versprechen sich von der Amputation eines Beines die Wiederherstellung ihrer Männlichkeit (overachievement) bzw. deren Bewahrung (Beinamputation statt Penisamputation). Den von Berger et al. (2005) beschriebenen Patienten hat die Amputation der Beine tatsächlich vor der Amputation seines Penis bewahrt. 
und Psychopharmaka helfen, das extrem geringe Selbstwertgefühl zu erhöhen, Krankheitseinsicht zu erreichen und andere Lebensentwürfe als Regression vorstellbar zu machen und ggf. die eigene Homosexualität zu akzeptieren.

2. Paraphilie: Ein Amputationswunsch kann aus einer sexuell motivierten Alloapotemnophilie resultieren, also aus einem Fetischismus für Amputationen, möglicherweise ausgelöst durch Stress oder Depression. Bei diesem Typ ist das Devotee-Verhalten vorherrschend, das ggf. in ein Pretender- und ein Wannabe-Verhalten umschlägt. ${ }^{93}$ Bei Alloapotemnophilie wird - wie bei anderen Paraphilien - meist keine Therapie gewünscht. Gefordert wird vielmehr gesellschaftliche Toleranz sowie ein leichter $\mathrm{Zu}$ gang zu den Objekten der Begierde. Ist mit der Alloapotemnophilie ein Leidensdruck verbunden, könnte eine geeignete Sexualtherapie hilfreich sein.

3. Body Dysmorphic Disorder und Zwangsstörung: Wie Menschen mit Body Dysmorphic Disorder leiden auch BIID-Patienten unter unkontrollierbaren, zwanghaften Gedanken über ihre äußere Erscheinung, und in beiden Fällen ist die Krankheitseinsicht meistens gering; vielmehr wird mit wahnhafter Intensität auf der Annahme des körperlichen Makels beharrt. Gekennzeichnet ist diese Störung mit einer zwanghaften gedanklichen, aber auch praktischen Beschäftigung mit dem als störend oder falsch empfundenen Körperteil. Korreliert ist diese Störung mit Zwangsstörungen, Depressionen, Soziophobie, Essstörungen, Substanzmissbrauch, Feindseligkeit und Suizidalität. Bei Patienten mit Body Dysmorphic Disorder sind chirurgische Eingriffe i. d. R. vergeblich. ${ }^{94}$ Mit Psychotherapie und Psychopharmaka lässt sich die Störung vielleicht lindern.

4. Hirnorganisch bedingte Störung des Körperbildes: Schließlich kann ein Amputationswunsch aus dem Gefühl, dass ein bestimmter Körperteil fremd und nutzlos ist, resultieren. Nur diese Störung ist eine Body Integrity Identity Disorder im eigentlichen Sinne. Sie ist höchstwahrscheinlich hirnorganischen Ursprungs und verwandt mit dem Fremde-Hand-Syndrom, das in seltenen Fällen nach einem Schlaganfall auftritt. Wie Körperbildstörungen nach rechtsseitigen Schlaganfällen ist auch BIID durch Anosognosia, also Uneinsichtigkeit in die Krankheit, gekennzeichnet. Nach der Hypothese von Ramachandran und McGeoch (2007) ist die Ursache von BIID eine funktionelle Störung im rechten Scheitellappen. Falls der Amputationswunsch auf einer der oben beschriebenen neurologischen Störungen basiert, könnten in Zukunft kausale Therapien möglich werden. Zunächst könnten neuropsychologische Rehabilitationsmaßnahmen gewisse Verbesserungen bringen. ${ }^{95}$ Wenn das hirnorganische Substrat des gestörten Teils des Körperbildes präzise ermit-

93 Beispiele hierfür sind die von Money/Jobaris/Furth (1977) beschriebenen Fälle der homosexuellen Amputationsfetischisten mit Amputationswünschen sowie der von Wise/Kalyanam (2000) geschilderte Fall des Amputationsfetischisten, der seinen eigenen Penis amputiert hat.

$94 \mathrm{Vgl}$. Crerand/Franklin/Sarwer (2006).

95 Vgl. auch Ramachandran/Blakeslee (2002), S. 57-83. 
telt werden kann, lässt sich dieses möglicherweise durch kontinuierliche Hirnstimulation durch implantierte Elektroden ${ }^{96}$ oder durch einen mikrochirurgischen oder radiochirurgischen Eingriff therapieren. Eine transkranielle Magnetstimulation könnte vielleicht zumindest zeitweiligen Erfolg bringen.

\section{Medizinethische Diskussion über Amputationen bei BIID}

Im Folgenden werde ich die medizinethischen Argumente pro und contra Amputation bei BIID-Patienten, die in der Debatte vorgebracht worden sind, darstellen und diskutieren und anschließend die Debatte über BIID innerhalb der Medizinethik skizzieren.

\subsection{Diskussion der Argumente pro Amputation bei BIID}

\section{Recht auf freie Gestaltung des eigenen Körpers}

Nach liberaler Auffassung hat jeder Mensch grundsätzlich das Recht, seinen Körper so zu gestalten, wie er es möchte. Freiwillige Amputationen stünden in einem Kontinuum mit anderen freiwillig herbeigeführten Körperveränderungen, z. B. mit Tätowierungen, Piercing, Schmucknarben, aufgespritzten Lippen, vergrößerten Brüsten und Designer-Vaginas. ${ }^{97}$

Die amerikanische Medizinethikerin Annemarie Bridy (2004) vertritt die Ansicht, dass Wunsch-Amputationen genauso wie kosmetische Operationen ein legitimes Mittel der Suche nach Glück und des Strebens nach Authentizität seien. Beide dienten dazu, den Körper dem persönlichen Idealbild anzupassen. Sie bewertet den Amputationswunsch nicht als irrational, nur weil er eine Behinderung bezwecke: Vielmehr sei dieser ein legitimer Wunsch nach einer Selbstmodifikation. Diese These begründet sie im Anschluss an Tom Koch (2001) mit dem Social Difference Model, nach dem „Behinderte“ nicht „behindert" sind (wie im Medical Model), sondern „anders“. ${ }^{98}$

Der Vergleich von Wunschamputationen mit Tätowierungen, Schmucknarben, Piercing etc. hinkt allerdings: Mit diesen demonstriert man seine Zugehörigkeit zu einer Gesellschaft bzw. Subkultur. Die damit verbundenen Verletzungen werden in Kauf genommen, um dauerhaft und gut sichtbar die Einsatz- und Opferbereitschaft für die Cruppe sowie die Abgrenzung von

96 Zur Tiefenhirnstimulation zur Behandlung psychiatrischer Krankheiten vgl. Aouizerate et al. (2004); Brentrup et al. (2004); Funkiewiez et al. (2003 und 2004); Müller (2007); Northoff (2001); Perozzo et al. (2001); Schneider et al. (2003); Tammer (2006); Witt et al. (2006).

97 Designer-Vaginas sind die neueste Mode der plastischen Chirurgie; dabei handelt es sich um die operative Herstellung einer abgeflachten Vulva, die dem kindlichen Aussehen von Models in Modezeitschriften bzw. digital veränderten Bildern aus Pornozeitschriften entsprechen soll. - Vgl. SPIEGEL Online, 25.05.2007.

98 Vgl. Bridy (2004), S. 152. - Nach Tom Koch (2001) ist die Welt der Behinderungstheorien (Disability Theories) in zwei Lager gespalten: Nach dem Medical Model ist eine Behinderung eine negative Variation der physischen Norm, die notwendigerweise nachteilig ist. Nach dem Social Difference Model entsteht Behinderung nur durch soziale Diskriminierung aufgrund einer Andersartigkeit. 
rivalisierenden Gruppen zu demonstrieren. ${ }^{99}$ Freiwillige Amputationen sind dagegen kein Konformitätsbeweis für eine Gesellschaft oder Subkultur.

Ebenso wenig schlüssig ist der Vergleich mit Brustvergrößerungen, Lippenaufspritzungen, Beinverlängerungen, Nasen- und Augenmodifikationen etc.: Mit diesen Maßnahmen wird eine Übereinstimmung mit dem aktuellen Schönheitsideal der Gesellschaft erstrebt, und sie sind Mittel im Konkurrenzkampf um begehrte Partner, Aufmerksamkeit, Einfluss, Macht und Geld. ${ }^{100}$

\section{Autonomie}

Die Patientenautonomie ist eins der wichtigsten Prinzipien der Medizinethik. Viele BIID-Patienten berufen sich auf das Autonomieprinzip, um die gewünschte Amputation durchzusetzen. ${ }^{101}$ Auch die Medizinethiker Baynes und Levy (2005) folgern aus dem Autonomieprinzip, dass ein wohldurchdachter, lang anhaltender Amputationswunsch eines nicht psychotischen Patienten nach angemessener Aufklärung über die Folgen einem Chirurgen die Amputation eines gesunden Körperteils erlaube. ${ }^{102}$

Das Prinzip Patientenautonomie darf aber nur angewandt werden, wenn ein Patient zu rationalen Entscheidungen in der Lage ist. Aus psychiatrischer Sicht sind BIID-Patienten aber, obwohl sie nicht psychotisch sind, in ihrer Rationalität und Autonomie eingeschränkt, und ihr Amputationswunsch ist

99 Dies lässt sich mit der evolutionspsychologischen „Theorie der teuren Rituale“ von Richard Sosis und Eric Bessler erklären: Für die meisten Rituale religiöser, aber auch anderer Gruppen werden Zeit und materielle Ressourcen verschwendet, und manche sind gesundheitsschädlich oder sogar lebensbedrohlich. Gerade die vordergründige Schädlichkeit der Rituale hat einen enormen Nutzen für den Zusammenhalt der Gruppe: „Die Grundlage jeder Gemeinschaft ist die erfolgreiche Zusammenarbeit aller ihrer Mitglieder [...]. Das Problem dabei: Aus der Sicht des Einzelnen ist es günstiger, die anderen schuften zu lassen und sich selbst zu schonen. Um die Kooperation aufrechtzuerhalten, bedarf es also sozialer Mechanismen, die das ,Trittbrettfahren` verleiden. [...] Wer [...] eine schmerzhafte Zeremonie vollzieht, kommuniziert unmissverständlich: ,Ich identifiziere mich mit unserer Gruppe und glaube an das, wofür sie steht." Vgl. Sosis (2005), S. $46 \mathrm{f}$.

100 Jordan (2004) hat den Diskurs des „Plastic Body“ kritisch analysiert. Er konstatiert, dass die plastischen Chirurgen erfolgreich die Vorstellung des menschlichen Körpers als einer plastischen Substanz etabliert hätten, die Chirurgen verändern könnten, und die man auch verändern lassen sollte, um die eigenen Körperbildideale zu realisieren. Das Modell des „Plastic Body“ basiere auf einem starken cartesianischen Dualismus und einer „mind over matter“-Perspektive: Demnach müsse der Körper dem idealisierten Selbstbild angepasst werden. In Wirklichkeit werde aber nicht jedes individuelle Körperidealbild von Chirurgen realisiert; vielmehr bestimmten Chirurgen und die übrige medizinische Community über den Zugang zur plastischen Chirurgie, und um diesen gewährt zu bekommen, müsse das angestrebte Körperbild dem idealen Körperbild der Mediziner entsprechen. Um plastische Operationen zu erhalten, sei es darüber hinaus nötig, im medizinisch akzeptierten Kontext zu argumentieren. Als legitimes Argument für chirurgische Körperveränderungen gelte, wenn ein Patient die „Falschheit“ seines Körpers plausibel machen könne, für die er eine chirurgische „Korrektur“ benötige, um sein psychisches Leiden unter dem falschen Körper zu therapieren. Während diese Argumentation bei Patienten, die sich durch Schönheitsoperationen dem anerkannten Schönheitsideal annähern wollen, prinzipiell erfolgversprechend sei, scheiterten Wannabees mit praktisch derselben Argumentation, da deren Vorstellung des „richtigen“ Körpers den Vorstellungen der Ärzte davon zu sehr widerspreche.

101 Beispielsweise konstatiert der von Everaerd (1983) beschriebene Patient, der unbedingt ein Holzbein haben wollte: „Only I am the master of my body [...].“ (S. 290). „Besides that I want it [the amputation] to happen (a freely made decision), and I will, without exception, accept all the foreseen consequences." (S. 291). Die Behauptung einer freien Entscheidung steht allerdings im Widerspruch zur Aussage des Patienten, er sei „completely controlled by it [the desire]“ (S. 288). - Vgl. auch Skatssoon (2005).

102 Vgl. Bayne/Levy (2005), S. 79-82. 
als zwanghafter Wunsch, nicht als freier Wunsch zu sehen. BIID-Patienten leiden zumindest an einer monothematic delusion, ${ }^{103}$ ähnlich wie Patienten mit Anorexie, Capgras-Syndrom oder Zählzwang. Da es sich hierbei um einen Wahn handelt, sind sie unfähig zur Krankheitseinsicht. Nicht nur, aber vor allem bei Patienten mit eingeschränkter Entscheidungsfreiheit ist das Autonomieprinzip durch die medizinethischen Prinzipien Benefizienz und NonMalefizienz einzuschränken. Einer Magersüchtigen den Magen zu verkleinern oder einem BIID-Patienten ein gesundes Bein zu amputieren widerspricht dem Prinzip Non-Malefizienz.

\section{Amputation als einzige wirksame Therapieoption}

Die Amputation bei BIID-Patienten wird vor allem damit gerechtfertigt, dass sie unter einer Störung leiden, die nur mittels Amputation therapierbar sei und damit erfolgreich. ${ }^{104}$ Weder Psychotherapie noch Psychopharmaka könnten den Amputationswunsch abstellen. ${ }^{105}$ Nach First (2004) ist BIID eine Identitätsstörung wie Gender Identity Disorder, und wahrscheinlich sei hier ebenfalls die Amputation die einzige Lösung. Robert Smith, der bei zwei BIID-Patienten je ein gesundes Bein amputiert hat, schließt sich dieser These an. ${ }^{106}$ Smith und Fisher rechtfertigen Amputationen bei BIID-Patienten außerdem mit der These, dass bei BIID der physische Körper und der wahrgenommene Körper nicht übereinstimmten, aber mittels Amputation in Übereinstimmung gebracht werden könnten. ${ }^{107}$

Außerdem sei die freiwillige chirurgische Entfernung gesunder Körperteile zum Zweck der Verbesserung des ,psychologischen Wohlergehens' bereits in drei Fällen etabliert:

1. kosmetische Chirurgie,

2. Lebendorganspende,

3. sex reassignment surgery.

Die Amputation zur Verbesserung des psychologischen Wohlergehens von BIID-Patienten könnte zu diesem Spektrum gehören. ${ }^{108}$

Dagegen spricht, dass auch die genannten Verfahren mit guten Argumenten kritisiert werden und teilweise ethisch problematisch sind. Evidenzbasierte Studien, die zeigen, dass kosmetische Operationen ohne medizinische Indikation sowie Operationen zur „Geschlechtsumwandlung“ bei Transsexuellen tatsächlich das psychische Wohlergehen nachhaltig verbessern und dass alle weniger invasiven Therapien erfolglos sind, fehlen bislang.

103 Dies räumen auch Bayne/Levy (2005), S. 80, ein.

104 Von den 52 von First befragten Patienten hatten 6 eine Amputation an der gewünschten Stelle und fühlten sich danach nach eigenem Bekunden besser als je zuvor und hatten keine weiteren Amputationswünsche. Vgl. First (2004), S. 8. - Diese Argumentation vertreten Bayne/Levy (2005), Bridy (2004), Furth/Smith (2000).

105 Vgl. First (2004), S. 8; Bayne/Levy (2005), S. 83; Braam et al. (2006), S. 36.

106 Vgl. Smith/Fisher (2003) und Fisher/Smith (2000).

$107 \mathrm{Vgl}$. Fisher/Smith (2000).

108 Johnston/Elliot (2002), S. 432, führen dieses Argument aus, argumentieren aber dagegen. 
Ebenso gibt es keine einzige Studie, die belegt, dass Amputationen eine geeignete Therapie für BIID sind, sondern nur einige anekdotische Berichte. Die Befürworter der Wunschamputationen stützen sich auf ungefähr zehn wissenschaftlich dokumentierte Fallbeispiele, deren Ergebnisse statistisch nicht signifikant sind, zumal die Auswahl der Beispiele wissenschaftlichen Standards nicht entspricht, da sie nur diejenigen Amputierten einbezieht, die Kontakt zu Medien und Forschern suchen. ${ }^{109}$ Dasselbe gilt auch für die im Internet verbreiteten Erfolgsgeschichten: Wie viele Fälle diesen gegenüber stehen, in denen die Patienten ihre freiwillige Amputation bereuen, liegt im Dunkeln. Außerdem fehlen wissenschaftliche Studien zur Wirkungslosigkeit der anderen Therapien; auch hier stützt sich die Argumentation auf wenige Einzelfallberichte gescheiterter (ggf. psychopharmakologisch unterstützter) Psychotherapien.

Grundsätzlich könnte eine Amputation höchstens Patienten mit einer Körperbildstörung Erleichterung verschaffen, während sie bei Patienten, die sich von einer Behinderung Aufmerksamkeit und Liebe versprechen, nur eine ungerechtfertigte Körperverletzung wäre. Aber selbst beim Vorliegen einer Körperbildstörung ist die Schlussfolgerung von Smith und Fisher nicht zwingend: Wenn der physische Körper und das Körperbild nicht übereinstimmen, kann eine Übereinstimmung grundsätzlich auf zwei Wegen erreicht werden: entweder durch Veränderung des physischen Körpers (also eine Amputation) oder durch eine Modifikation des Körperbilds. Dieses wird vom Gehirn generiert; um es zu modifizieren, ist also eine Veränderung des Gehirns erforderlich: durch neuropsychologische Rehabilitationsmaßnahmen, funktionelle Neurochirurgie oder transkranielle Magnetstimulation. Es stellt sich dann die Frage, ob ein Eingriff in das Gehirn oder die Amputation einer Gliedmaße ethisch problematischer ist. Für den Eingriff in das Gehirn spricht bei BIID, dass die Störung tatsächlich im Gehirn und nicht im Arm oder Bein lokalisiert ist. Eine Therapie des Gehirns wäre eine ursächliche Therapie, eine Amputation dagegen ein Kurieren eines Symptoms.

Untersuchungen mit funktioneller Bildgebung (fMRT oder PET) sind bei BIID-Patienten noch nicht durchgeführt worden, sondern lediglich einige strukturelle Gehirnuntersuchungen, die allerdings keinen konkreten Hinweis erbracht haben. Solange die schon heute verfügbaren diagnostischen Möglichkeiten nicht einmal ausgeschöpft worden sind, sollte nicht voreilig amputiert werden, um den Patienten keine nicht wieder gutzumachenden Schäden zuzufügen. Und selbst wenn mit den derzeitigen Geräten keine funktionellen oder strukturellen Gehirnschäden bei BIID-Patienten nachweisbar sein sollten, heißt dies nicht, dass solche nicht vorliegen. Möglicherweise reicht die Auflösung der heute verfügbaren Geräte nicht aus, um die Ursache von BIID zu identifizieren. Nichtsdestotrotz sollten dann m. E. die Ergebnisse weiterer Forschung mit höher auflösenden Geräten abgewartet werden, statt die Patienten für den Rest ihres Lebens zu verstümmeln. 


\section{Amputation durch Chirurgen besser als Do-it-yourself-Amputation}

Einige BIID-Patienten unternehmen lebensgefährliche Versuche, um den Körperteil, der Gegenstand ihrer Fixierung ist, so zu verletzen, dass ein Chirurg ihn amputieren muss, oder sogar, ihn selbst abzutrennen. Sie verwenden dazu Gewehre, Kettensägen, Hämmer, Trockeneis, Schnürriemen etc. ${ }^{110}$ Manche infizieren die Wunden mit Darm- oder Eiterbakterien. ${ }^{111}$ Für von Chirurgen durchgeführte Amputationen spricht, dass eine sachgemäß durchgeführte Operation immer noch besser ist als vom Patienten durchgeführte Amputationsversuche, die bereits zu Todesfällen geführt haben. ${ }^{12}$ Bayne und Levy schließen daraus: „Surgery might be the least of all evils.“"113

Dieses Argument impliziert, dass die Amputation unausweichlich sei, und sich nur die Frage stelle, wer sie wie durchführt. Auf wie viele Wannabes dies zutrifft, ist nicht bekannt; wahrscheinlich nur auf eine Minderheit. Gegen dieses Argument spricht, dass das Angebot ordnungsgemäß durchgeführter Amputationen auch solche BIID-Patienten anziehen könnte, die davor zurückschrecken, sich selbst lebensgefährlich zu verletzen.

\section{Weitere Amputationen für die Forschung erforderlich}

Smith und Fisher stellen fest, dass der mögliche Nutzen der verschiedenen Therapien nur bewertet werden könne, wenn weitere Operationen sowie andere Behandlungen durchgeführt und verglichen werden. ${ }^{114}$ Annemarie Bridy fordert sogar, medizinische Heilversuche ohne wissenschaftliche Basis an Patienten, denen keine bewährte Therapie angeboten werden kann, in Provinzkrankenhäusern durchzuführen - selbst außerhalb klinischer Studien und ohne Forschungsprotokoll. ${ }^{115}$

Die Forderung Bridys widerspricht nicht nur den Regeln guter Wissenschaft, sondern ist auch ethisch höchst bedenklich, was auch für Smiths und Fishers Argument gilt. Gerade so schwerwiegende, irreversible Eingriffe wie Amputationen, die lebenslange gesundheitliche, finanzielle und soziale Belastungen nach sich ziehen, müssen besonders strikten wissenschaftlichen und medizinethischen Ansprüchen genügen. Riskante Heilversuche lassen sich ggf. rechtfertigen, wenn sie der Lebensrettung oder der Heilung schwerer Krankheiten dienen und keine bewährte Therapieoption vorhanden ist. Dies ist aber bei BIID-Patienten nicht der Fall. Völlig inakzeptabel sind die von Smith durchgeführten Experimente unter Umgehung der Ethikkommission und Missachtung wissenschaftlicher Standards.

110 Vgl. Sorene et al. (2006), S. 593; First (2004); Everaerd (1983); Furth/Smith (2000), S. 88.

$111 \mathrm{Vgl.} \mathrm{Bensler/Paauw} \mathrm{(2003);} \mathrm{Storm/Weiss} \mathrm{(2003).}$

112 Vgl. Smith/Fisher (2003), S. 188; Bridy (2004), S. 148.

113 Vgl. Bayne/Levy (2005), S. 79.

$114 \mathrm{Vgl.Smith} /$ Fisher (2003).

115 Vgl. Bridy (2004), S. 155. 


\subsection{Diskussion der Argumente contra Amputation bei BIID}

\section{Komplikationen, Nebenwirkungen und Folgebehandlungen von Amputationen}

Amputationen sind schwerwiegende Eingriffe, die schwere Komplikationen und langfristige Nebenwirkungen haben können: starke Nachblutungen, Einblutungen in das Gewebe, Infektionen, Druckschäden an den Nerven oder Weichteilen mit Empfindlichkeitsstörungen, Hautschäden, in seltenen Fällen Lähmungen. Aufgrund von Über- und Fehlbelastung können Funktionsstörungen, Versteifungen und Verkrümmungen in der Restgliedmaße oder der Cliedmaße der Gegenseite entstehen. Zur Vorbeugung ist eine frühzeitige Bewegungsbehandlung durch Krankengymnasten erforderlich. Durch Prothesen kann es zu Druckgeschwüren bis zu Nekrosen kommen, was manchmal Nachamputationen erforderlich macht. Phantomschmerzen sind sehr häufig; sie erfordern eine umfangreiche Schmerzbehandlung durch Spezialisten. Die abgetrennten Nerven an der Amputationsstelle bilden in seltenen Fällen schmerzhafte Nervenwucherungen und Knoten (Amputationsneurome), die chirurgisch entfernt werden müssen. Zwischen den großen Arterien und Venen kann es, vor allem nach Beinamputationen, zu einer Kurzschlussverbindung (Shunt) kommen, die chirurgisch unterbunden werden muss. Durch Maßnahmen zur Ruhigstellung können Thrombosen entstehen, die zur Embolie führen können. Die vorbeugende Gabe von gerinnungshemmenden Mitteln (z.B. Heparin) kann wiederum zur Blutungsneigung und schwerwiegenden Störungen der Blutgerinnung führen. Infektionen durch Fremdblutkonserven mit Hepatitis-Viren, HIV oder Erregern der neuen Variante der Creutzfeldt-Jakob-Erkrankung sind nicht sicher ausschließen. ${ }^{116}$ Durch antibiotikaresistente Keime, die sich in vielen Krankenhäusern ausbreiten, besteht außerdem ein Risiko tödlicher Blutvergiftungen.

\section{Psychische Belastungen durch Amputationen}

Für die meisten Menschen ist eine Amputation ein traumatisches Ereignis, unter dessen Folgen sie ihr Leben lang leiden. Meist geht einer Amputation entweder eine schwere Erkrankung voraus, z. B. Diabetes, Knochenkrebs, Wundinfektionen, Erfrierungen, Osteomyelitis, oder schwere Verletzungen durch Unfälle, Schussverletzungen, Verletzungen durch Minen oder Komplikationen nach einer Operation. ${ }^{17}$

Die psychischen Folgen von Amputationen an den unteren Extremitäten haben Suada Kapidžić-Duraković et al. aus Bosnien-Herzegowina untersucht, sowohl an Veteranen mit kriegsbedingten Amputationen als auch an Zivilisten mit krankheitsbedingten Amputationen. Dabei haben sie festgestellt, dass eine Amputation nicht nur den Verlust eines Körperteils bedeutet, sondern auch den Verlust der körperlichen Identität, und schwerwiegende psychische Folgen hat: Die meisten Patienten mit Amputationen zeigen signifikant er-

116 www.chirurgie-portal.de/orthopaedie/arm-bein-amputation.html.

117 Vgl. Wallace (2005). - In den USA ist Diabetes die wichtigste Ursache für nicht-verletzungsbedingte Amputationen. 
höhte Werte von Empfindlichkeit, Ängstlichkeit, Feindseligkeit und Paranoia. ${ }^{118}$ Bei Patienten, die wegen Krankheiten oder Unfällen Amputationen erleiden, sind vor allem Depressionen häufig. ${ }^{119}$

Ob diese Befunde auf BIID-Patienten übertragbar sind, ist eine offene Frage, da diese die Amputation nicht gegen ihren Willen erleiden. Die anekdotischen Berichte von freiwillig Amputierten über ihr glückliches Leben nach der Amputation sind zwar wissenschaftlich mit großer Vorsicht zu betrachten, dennoch müssen sie Zweifel an dem Argument psychischer Schäden durch eine freiwillige Amputation hervorrufen.

\section{Symptomverschiebung}

In manchen Fällen verlagert sich die Störung nach einer Amputation auf einen anderen Körperteil. ${ }^{120}$ Einige Patienten verstümmeln nacheinander verschiedene Gliedmaßen, so dass diese amputiert werden müssen. ${ }^{121}$ Sorene et al. (2006) warnen daher, die Amputation als Therapie für BIID anzusehen, da die anfängliche Zufriedenheit damit durchaus keine lebenslange Erlösung bedeuten muss, sondern die Forderung weiterer Amputationen nach sich ziehen kann. Unter Umständen ist erst Schluss, wenn alle vier Gliedmaßen amputiert sind.

Bei Patienten, bei denen ein bestimmter Körperteil nicht in das Körperbild integriert werden kann, ist eine Fortsetzung des Amputationswunsches allerdings unwahrscheinlich.

\section{Grundsätzlich keine Unterstützung für gesundheitsgefährdende Körperveränderungen}

Nach dem Non-Malefizienz-Prinzip sollten Ärzte grundsätzlich keine gesundheitsschädlichen Eingriffe vornehmen. Dass manche Ärzte heutzutage gesundheitsschädliche Operationen wie Brustvergrößerungen durchführen, kann keine Rechtfertigung für andere, noch invasivere gesundheitsschädliche Eingriffe sein, zumal an Patienten mit psychiatrischen Störungen. WunschAmputationen bei BIID-Patienten sollten aus denselben Cründen wie Fettabsaugungen oder operative Magenverkleinerungen bei Magersüchtigen nicht durchgeführt werden. ${ }^{122}$

118 Vgl. Kapidžić-Duraković et al. (2006).

119 Vgl. Wallace (2005), S. 316-318.

120 Beckford-Ball (2000), S. 188.

121 Ein Beispiel ist der von Sorene et al. (2006) beschriebene Patient, der erst sein rechtes Bein nach Selbstverletzung und Infektion amputieren ließ, außerdem mehrere Finger amputierte und schließlich seine linke Hand mit einer Axt abhackte. Ein weiteres Beispiel ist der von Berger et al. (2005) beschriebene Transsexuelle, der nach erfolgter Beinamputation zwar auf die Amputation seiner Genitalien verzichtete, da er sich wieder als Mann betrachtete, aber über die Amputation eines Armes nachdenkt, seit er einen Mann kennt, dem alle vier Gliedmaßen fehlen.

122 Vgl. auch Beckford-Ball (2000), S. 188, der sich hier auf Munro (2000) bezieht. 


\section{Ökonomische Konsequenzen}

Nach einer Berechnung von Richard Alexander (2003) betragen die Folgekosten für eine Amputation eines Beines unterhalb des Knies, die für eine gute Versorgung erforderlich wären, 105.000 \$ pro Jahr. Darin enthalten sind nur die Kosten für medizinische Dienstleistungen, Haushaltshilfen, Spezialausrüstung und Umbauten in der Wohnung und am Auto. Ggf. verlorenes Arbeitseinkommen ist darin noch nicht berücksichtigt. Auf den Sozialstaat kommen durch Amputationen enorme Kosten zu, da die Betreffenden Anspruch auf Frührente, Sozialhilfe, Krankenversicherung, Rehabilitationsmaßnahmen etc. haben. Die Operationskosten von ca. 10.00o € sind auf jeden Fall nur der geringste Teil der Kosten.

\section{BIID als neue „Modekrankheit“ und mögliche Identität für Regressive}

Wenn BIID als eine neue Störung im DSM-V klassifiziert würde, könnte es schnell zu einer neuen „Modekrankheit“ werden. Gerade psychiatrische Kategorien haben häufig einen „Looping-Effekt“, der manche Menschen veranlasst, sich durch diese eine Identität zu konstruieren. Ein Beispiel dafür ist Multiple Persönlichkeit (Dissoziative Persönlichkeitsstörung). ${ }^{123}$ Neue Krankheitsdiagnosen entwickeln sich mit der Formulierung einer wissenschaftlichen Definition, von institutionellen Strukturen zu ihrer Diagnose und Behandlung, formalen Behandlungsrichtlinien, Messskalen und einer formalen Aufnahme in DSM und ICD. Wenn BIID erst einmal als formale psychiatrische Störung anerkannt ist, werden sich rasch entsprechende institutionelle Strukturen entwickeln. So wie Transsexualität noch in den 1950er Jahren als extrem seltene Störung galt, wurde sie in den 1970er Jahren schon als nicht ungewöhnliche Geschlechtsstörung beschrieben, und heute kommt allein ein Chirurg in Colorado auf über 4.000 sog. sex reassignments. ${ }^{124}$ Die Anerkennung chirurgischer Eingriffe als geeignete Therapie für psychiatrische Erkrankungen, wie im Fall der sog. geschlechtsanpassenden Operationen bei Transsexuellen, erzeugt unmittelbar einen Markt für entsprechende chirurgische und sonstige medizinische Dienstleistungen. Koalitionen aus finanziell interessierten Ärzten und Kliniken sowie den Lobbygruppen der Transsexuellen haben in vielen Ländern, u. a. in Deutschland, sogar erreicht, dass die gesetzlichen Krankenkassen die enormen Kosten für „geschlechtsumwandelnde“ Operationen und dadurch bedingte Folgeschäden sowie das Krankentagegeld übernehmen müssen.

Bayne und Levy bezweifeln, dass BIID zu einer Modekrankheit wird, weil Amputationen dem gängigen Körperideal zu sehr zuwiderlaufen. ${ }^{125}$ Das ist zweifellos richtig; BIID könnte aber für Menschen mit starken regressiven Tendenzen eine attraktive Option darstellen, wenn es eine anerkannte Störung werden sollte, für deren Folgen die Versicherungen bezahlen müssten.

123 Zum Looping effect of human kinds vgl. Hacking (1995), S. 239. - Diese Befürchtung äußern Johnston/Elliot (2002), S. 434, sowie Bayne/Levy (2005), S. 85.

124 Vgl. Johnston/Elliot (2002), S. 434.

$125 \mathrm{Vgl}$. auch Bayne/Levy (2005), S. 85. 
Menschen, die ohnehin den gesellschaftlichen Idealen nicht entsprechen können - weder vom Aussehen noch von der Leistungsfähigkeit - hätten als Amputierte zugleich ein Alibi und ein Einkommen.

\section{Slippery Slope}

Jason Beckford-Ball (200o) führt aus, dass Amputationen gesunder Cliedmaßen unterbleiben sollten, weil dies sonst zu einer Akzeptanz von Selbstverletzungen führen könnte, ohne dass die zugrunde liegenden psychologischen Ursachen behandelt würden: „It is a case where the few must suffer to safeguard the majority. ${ }^{\text {"126 }}$

Dieses Argument ist gesinnungsethisch. Zwar besteht hier wie in vielen anderen Fällen die Gefahr eines Slippery Slope, doch bei sorgfältiger Indikationsstellung lässt sich die Ausweitung der Akzeptanz einer höchstens im Einzelfall akzeptablen Maßnahme i.d. R. vermeiden. Das Leiden eines Individuums in Kauf zu nehmen, um gesellschaftliche Normen zu schützen, ist m. E. nicht zu rechtfertigen.

\section{Freiwillige Amputationen sind ein Hohn für Kriegsversehrte und Kranke}

Vor allem durch Kriege erleiden Menschen Verletzungen, die zu einer Amputation führen. In islamischen Ländern werden etlichen Menschen wegen der Scharìa die Hände amputiert. Zahlreiche Menschen müssen sich außerdem wegen Krankheiten, insbes. Diabetes, sowie nach Unfällen Amputationen unterziehen. Bei der Diskussion über die von BIID-Patienten gewünschten Amputationen sollte man das Leid der Opfer von Krieg, Gewaltherrschaft, Unfällen und Krankheiten nicht ganz aus den Augen verlieren, denen ,freiwillige“"Amputationen wie Hohn erscheinen könnten. Daraus lässt sich schließen, dass Amputationen gesunder Körperteile mit Rücksicht auf diese Menschen verboten werden sollten. ${ }^{127}$

Auch dieses Argument ist gesinnungsethisch. Es würde gerade dann nicht zutreffen, wenn die betreffenden Körperteile von BIID-Patienten tatsächlich nicht gesund sein sollten, weil ihre neuronale Repräsentation gestört ist. ${ }^{128}$

\subsection{Kontroverse in der Medizinethik}

Die wenigen Stellungnahmen von Medizinethikern zu BIID sind höchst kontrovers und lassen sich in zwei Lager einteilen:

Zu den Gegnern der Wunschamputationen gehört Arthur Caplan, der Direktor des Bioethik-Zentrums der Universität von Pennsylvania. Er erklärt, dass solche Amputationen den Hippokratischen Eid auf das Extremste verletzen würden:

126 Beckford-Ball (2000), S. 188.

127 Ein Abgeordneter des schottischen Parlaments, Dennis Canavan, nannte die von Smith durchgeführten Amputationen „obszön“. Vgl. Bayne/Levy (2005), S. 85; Bridy (2004), S. 148 und 156.

$128 \mathrm{Vgl.} \mathrm{Bayne/Levy} \mathrm{(2005),} \mathrm{S.} 85$. 
„It's meshugeneh - absolutely nuts. It's absolute, utter lunacy to go along with a request to maim somebody. The cure is not to yield to the illness and conform to the obsession. And this is not just about, do no harm'. It's also about whether (sufferers) are competent to make a decision when they're running around saying, ,Chop my leg off. ‘'“29

Auch Erich H. Loewy, emeritierter Medizinprofessor und Founding Alumni Association Chair of Bioethics Associate der Philosophy University of California, äußert sich sehr ablehnend zu Wunschamputationen. ${ }^{130}$

Auf der anderen Seite stehen Befürworter der Wunschamputationen, die die Positionen der BIID-Protagonisten und des Chirurgen Robert Smith übernommen haben, z. B. die amerikanische Medizinethikerin Annemarie Bridy (2004): Sie stellt Amputationen gesunder Körperteile in ein Kontinuum mit kosmetischen Operationen und hält diese wie jene für legitime Mittel der Suche nach Glück und des Strebens nach Authentizität. Beide dienten dazu, den Körper dem persönlichen Idealbild anzupassen. Der Amputationswunsch sei kein irrationaler Wunsch nach einer Behinderung, sondern ein legitimer Wunsch nach einer Selbstmodifikation.

Thomas Schramme (2006) befindet ebenfalls, dass „auch die freiwillige Verstümmelung als eine Form der wertneutralen Körpermodifikation gelten“ müsse. Dies schränkt er allerdings auf Fälle ein, bei denen „grundlegende Fähigkeiten wie Mobilität oder Wahrnehmung " nicht zerstört werden, da diese „notwendige Bestandteile einer autonomen Lebensweise“ seien. ${ }^{131}$

Der Philosoph Floris Tomasini (2006) übernimmt bedingungslos die Position der BIID-Aktivisten und des Chirurgen Robert Smith. Seine weitreichenden Folgerungen stützt er ausschließlich auf die BBC-Fernsehdokumentation und das Buch von Gregg Furth und Robert Smith (2000). Die Fachliteratur zu BIID von Psychiatern und Neurologen ignoriert er komplett. Den Ethikern, die Selbstverstümmelung ablehnen, insbesondere Kant und den Kantianern, wirft er eine naturalistische Haltung bezüglich des Körpers vor - eine befremdliche Argumentation, da Kant bekanntlich die Pflichten bezüglich des eigenen Körpers transzendentalphilosophisch und nicht naturalistisch begründet hat. ${ }^{132}$ Außerdem spricht Tomasini den Gegnern der Wunsch-Amputationen Empathie ab; zum Verständnis des Amputationswunschs seien eine „deeper form of understanding“ und „some sort of empathetic recognition of what it is

129 Caplan, zitiert nach Dotinga (2000).

130 MedEthik-list, Loewy, 24.11.2005: „Wie nennt man einen Patienten, der sich ohne sichtbaren medizinischen Grund ein Bein abschneiden lassen will, nachdem es abgeschnitten wurde? Sicherlich ist er behindert, und je mehr er abschneiden lässt, desto behinderter wird er. Kriegt er dann Fürsorge? Persönlich finde ich die Diskussion wenigstens merkwürdig. Es scheint ein psychiatrisches Problem zu sein, wo wir Andern wenig oder nichts zu sagen können. [...] Solche Leute kämen - würde ich hoffen - in eine Anstalt oder ein Gefängnis. In einer Zeit, wo es in vielen Weltteilen (inkl. der USA) von Armen und Hungrigen wimmelt, wo man über Beschränkung des Gesundheitswesen spricht, und in den USA ein riesiger Prozentsatz keinen Zugang hat oder nur einen Zugang mit unerschwinglichem ,co-payment', sollte man sich vorstellen, dass es wichtigere Dinge zu diskutieren gibt Medizinethik ist nicht für unsere Belustigung geschaffen. [...] falls es [BID] psychiatrisch krank ist, so wird es kaum dadurch, dass man ein Bein abschneidet, geheilt werden." [Tippfehler korrigiert.]

131 Schramme (2006), S. 176.

132 Zu Kants Selbstverstümmelungsverbot nimmt auch Schramme (2006), S. 170-173, kritisch Stellung. 
to be wholly human “ erforderlich. ${ }^{133}$ Wie ein tieferes Verständnis bei gleichzeitiger Ignoranz gegenüber empirischen Fakten erwachsen soll, bleibt unklar.

Alexander Kissler befürchtet, dass die Amputationsbefürworter in der Medizinethik die Oberhand gewinnen werden:

„Und die ethische Großwetterlage scheint den BIIDlern günstig gesonnen. ,RollstuhL_jetzt‘ notiert: , Das ethische Verbot [der Wunsch-Amputation] ist nicht haltbar. Ich bin mir sicher, dass die analytische Philosophie der angelsächsischen Kultur früher oder später diese Antwort geben wird. “'134

Tatsächlich bewegen sich Schramme, Tomasini und Bridy im antinaturalistischen Mainstream der zeitgenössischen Philosophie, ersterer im Rahmen der analytischen Philosophie, letztere im Rahmen des Kulturrelativismus und der Disability Studies. Alle drei stützen ihre Argumentation auf den Begriff der Autonomie.

Der Begriff der Autonomie wird dabei allerdings missbraucht, um zwanghafte, selbstschädigende Wünsche zu autonomen Entscheidungen zu adeln. Man muss kein Anhänger neurowissenschaftlicher Erklärungsansätze menschlichen Wollens und Handelns sein, um bestimmte Wünsche als nicht rational, unfrei und zwanghaft zu klassifizieren - auch die Analytische Philosophie trifft diese Unterscheidung. ${ }^{135}$ Patienten, die krankheitsbedingt keine Krankheitseinsicht haben, eignen sich weder als noch zu entdeckende Cruppe Diskriminierter noch als Paradigma eines abstrakten Autonomie-Diskurses.

\section{Fazit}

BIID bzw. Apotemnophilie ist eine psychiatrische, wahrscheinlich hirnorganisch bedingte Störung. Die Amputation gesunder Körperteile wäre nur ein Kurieren an einem Symptom statt einer ursächlichen Therapie - eine solche kann nur im Gehirn ansetzen, sei es mit neuropsychologischen, psychopharmakologischen oder neurochirurgischen Methoden. Selbst wenn Amputationen eine subjektive Leidensminderung bringen sollten, sind sie aus medizinethischer Sicht nicht zu befürworten, da sie dem Patienten und ggf. seinem sozialen Umfeld erhebliche irreversible Schäden zufügen - höchstwahrscheinlich unnötigerweise, da vermutlich schon bald die hirnorganischen Ursachen von BIID identifiziert und in der Folge kausal behandelbar werden. Das Ziel der Therapie von BIID sollte nicht nur Leidensfreiheit sein, sondern Leidensfreiheit plus Behinderungsfreiheit: Statt das Leiden an einem als fremd empfundenen Körperteil einfach durch dessen Amputation zu beenden, sollte dieser Körperteil in das Körperbild integriert werden. Für dieses anspruchsvolle Ziel bedarf es High-Tech-Medizin; damit diese den Patienten helfen kann, dürfen sie nicht zuvor verstümmelt worden sein.

133 Vgl. Tomasini (2006), S. 110.

134 Vgl. Kissler (2005a).

135 Vgl. z. B. Bieri (2003). 


\section{Literatur}

Ach/Pollmann (2006): Johann S. Ach, Arnd Pollmann (Hrsg.): no body is perfect. Baumaßnahmen am menschlichen Körper - Bioethische und ästhetische Aufrisse, Bielefeld 2006

Alexander (2003): Richard Alexander, Lifecare Planning for the BK Amputee: Future Medical Costs, http:// consumerlawpage.com/article/amputee.shtml, Copyright: 2003 [05.02.2007]

American Psychiatric Association (2000): American Psychiatric Association, Diagnostic and statistical manual of mental disorders, Fourth Edition, Washington 2000 (DSM-IV)

Aouizerate et al. (2004): B. Aouizerate et al., Deep brain stimulation of the ventral caudate nucleus in the treatment of obsessive-compulsive disorder and major depression. Case report, Journal of Neurosurgery 101 (2004), 4, p. 682-686

Bayne/Levy (2005): Tim Bayne, Neil Levy, Amputees By Choice: Body Integrity Identity Disorder and the Ethics of Amputation, Journal of Applied Philosophy 22 (2005), 1, p. 75-86

Beckford-Ball (2000): Jason Beckford-Ball, The amputation of healthy limbs is not an option, British Journal of Nursing 9 (2000), 4, p. 188

Bensler/Paauw (2003): I. Mike Bensler, Douglas S. Paauw, Apotemnophilia masquerading as medical morbidity, Southern Medical Association Journal 96 (2003), 7, p. 674-676

Berger et al. (2005): Bertrand D. Berger, Jon A. Lehrmann, Gunnar Larson, Luca Alverno, Carol I. Tsao, Nonpsychotic, nonparaphilic self-amputation and the internet, Comprehensive Psychiatry 46 (2005), p. 380-383

Bieri (2003): Peter Bieri, Das Handwerk der Freiheit. Über die Entdeckung des eigenen Willens, Frankfurt 2003 Biran/Chatterjee (2004): Iftah Biran, Anjan Chatterjee, Alien Hand Syndrome, Archives of Neurology 61 (2004), 1, p. 292-294

Braam et al. (2006): Arjan W. Braam, Sako Visser, Daniëlle C. Cath et al., Investigation of the Syndrome of Apotemnophilia and Course of a Cognitive-Behavioural Therapy, Psychopathology 39 (2006), p. 32-37

Brentrup et al. (2004): Angela Brentrup, P. Ohrmann, M. Weckesser et al., Alterations of sociomoral judgement and glucose utilization in the frontomedial cortex induced by electrical stimulation of the subthalamicus nucleus (STN) in Parkinsonian patients, Meeting abstract, 55. Jahrestagung der Deutschen Gesellschaft für Neurochirurgie e. V. 2004, www.egms.de/de/meetings/dgnc2004/04dgnc0207.shtml

Bridy (2004): Annemarie Bridy, Confounding Extremities: Surgery at the Medico-ethical Limits of Self-Modification, Journal of Law, Medicine \& Ethics 32 (2004), 1, p. 148-158

Bruno (1997): Richard L. Bruno, Devotees, Pretenders and Wannabes: Two Cases of Factitious Disability Disorder, Journal of Sexuality and Disability 15 (1997), 4, p. 243-260

Crerand/Franklin/Sarwer (2006): Candice E. Crerand, Martin E. Franklin, David B. Sarwer, Body dysmorphic disorder and cosmetic surgery, Plastic and Reconstructive Surgery 118 (2006), 7, p. 167 e-80 e

Dotinga (2000): Randy Dotinga, Out on a limb, http://archive.salon.com/health/feature/2000/08/29/ amputation/index.html [02.03.2007]

Dudzinski (2005): Denise M. Dudzinski, „Amputate my arm please - I don’t want it anymore“, The Journal of Clinical Ethics 16 (2005), 3, p. 196-201

Dyer (2000): Clare Dyer, Surgeon amputated healthy legs, British Medical Journal 320 (2000), p. 332

Dyl et al. (2006): Jennifer Dyl, Jennifer Kittler, Katharine A. Phillips et al., Body dysmorphic disorder and other clinically significant body image concerns in adolescent psychiatric inpatients: prevalence and clinical characteristics, Child Psychiatry and Human Development 36 (2006), p. 369-382

Everaerd (1983): Walter Everaerd, A Case of Apotemnophilia: A Handicap as Sexual Preference, American Journal of Psychotherapy 37 (1983), 2, p. 285-293

First (2004): Michael B. First, Desire for amputation of a limb: paraphilia, psychosis, or a new type of identity disorder, Psychological Medicine 34 (2004), p. 1-10

Fisher/Smith (2000): Keren Fisher, Robert Smith, More work is needed to explain why patients ask for amputation of healthy limbs, Letters, British Medical Journal 320 (2000), p. 1147

Funkiewiez et al. (2003): A. Funkiewiez et al., Acute psychotropic effects of bilateral subthalamic nucleus stimulation and levodopa in Parkinson's disease, Movement disorders 18 (2003), 5, p. 524-530 
Funkiewiez et al. (2004): A. Funkiewiez, C. Ardouin, E. Caputo et al., Long term effects of bilateral subthalamic nucleus stimulation on cognitive function, mood, and behaviour in Parkinson's disease, Journal of Neurology, Neurosurgery and Psychiatry 75 (2004), 6, p. 834-839

Furth/Smith (2000): Gregg M. Furth, Robert Smith, Apotemnophilia: Information, Questions, Answers, and Recommendations about Self-Demand Amputation, Bloomington 2000

Hacking (1995): Ian Hacking, Rewriting the Soul: Multiple Personality and the Sciences of Memory, Princeton 1995

Johnston/Elliott (2002): Josephine Johnston, Carl Elliott, Healthy limb amputation: ethical and legal aspects, Clinical Medicine 2 (2002), 5, p. 431-435

Jordan (2004): John W. Jordan, The Rhetorical Limits of the „Plastic Body“, Quarterly Journal of Speech 90 (2004), 3, p. 327-358

Kapidžić-Duraković et al. (2006): Suada Kapidžić-Duraković, Azra Karabegović, Emir Halilbegović et al., Check list of symptoms SCL-90-R at persons with extremities amputations, Bosnian Journal of Basic Medical Sciences 6 (2006), 1, p. 58-61

Kissler (2005 a): Alexander Kissler, Mein Bein gehört mir. Weg damit!, Süddeutsche Zeitung 16.11.2005

Kissler (2005b): Alexander Kissler, Mein Haus, mein Auto, meine Schwerstbehinderung, Süddeutsche Zeitung 21.11.2005

Koch (2001): Tom Koch, Disability and difference: balancing social and physical constructions, Journal of Medical Ethics 27 (2001), p. 370-376

Lawrence (2006): Anne A. Lawrence, Clinical and theoretical parallels between desire for limb amputation and gender identity disorder, Archives of Sexual Behavior, 35 (2006), 3, p. 263-78

Lurija (1993): Alexander R. Lurija, Romantische Wissenschaft. Forschungen im Grenzbereich von Seele und Gehirn, Reinbek 1993

Merzenich et al. (1984): Michael M. Merzenich, Randall J. Nelson, Michael P. Stryker et al., Somatosensory cortical map changes following digit amputation in adult monkeys, The Journal of Comparative Neurology 224 (1984), 4, p. 591-605

Money/Jobaris/Furth (1977): John Money, Russell Jobaris, Gregg Furth, Apotemnophilia: Two Cases of SelfDemand Amputation as a Paraphilia, Journal of Sex Research 13 (1977), 2, p. 115-125

Müller (2007): Sabine Müller, Dilemmata bei operativen Eingriffen in das Gehirn, in: Dominik Groß, Sabine Müller (Hrsg.), Sind die Gedanken frei? Die Neurowissenschaften in Geschichte und Gegenwart, Berlin 2007, S. 175-207

Munro (2000): R. Munro, Disturbed patients have healthy limbs amputated. Nursing Times 96 (2000), 6, p. 25

Northoff (2001): Georg Northoff, Personale Identität und operative Eingriffe in das Gehirn. Neurophilosophische, empirische und ethische Untersuchungen, Paderborn 2001

Ovadia (2006): Daniela Ovadia, Gelinkt!, Gehirn \& Geist 12 (2006), S. 64-66

Pappalardo et al. (2004): A. Pappalardo, M. R. Ciancio, E. Reggio et al., Posterior Alien Hand Syndrome: Case Report and Rehabilitative Treatment, Neurorehabilitation and Neural Repair 18 (2004), p. 176-181

Perozzo et al. (2001): P. Perozzo, M. Rizzone, B. Bergamasco et al., Deep brain stimulation of subthalamic nucleus: behavioural modifications and familiar relations, Neurological Sciences 22 (2001), 1, p. $81 \mathrm{f}$.

Poeck/Hacke (1998): Klaus Poeck, Werner Hacke, Neurologie, Berlin et al., 10. Aufl., 1998

Ramachandran/Blakeslee (2002): Vilayanur Ramachandran, Sandra Blakeslee, Die blinde Frau, die sehen kann. Rätselhafte Phänomene unseres Bewusstseins, Reinbek 2002

Ramachandran/McGeoch (2007): Vilayanur Ramachandran, Paul McGeoch, Can vestibular caloric stimulation be used to treat apotemnophilia?, Medical Hypotheses 2007 Feb 8 [Epub ahead of print]

Sacks (2004): Oliver Sacks, Der Tag, an dem mein Bein fortging, Reinbek, 14. Aufl., 2004 [engl. Original: 1984]

Scepkowski/Cronin-Golomb (2003): Lisa A. Scepkowski, Alice Cronin-Golomb, The Alien Hand: Cases, Categorizations, and Anatomical Correlates, Behavioral and Cognitive Neuroscience Reviews 2 (2003), p. 261-277

Schneider et al. (2003): Frank Schneider, Ute Habel, Jens Volkmann et al., Deep Brain Stimulation of the Subthalamic Nucleus Enhances Emotional Processing in Parkinson Disease, Archives of General Psychiatry 60 (2003), p. 296-302

Schramme (2006): Thomas Schramme, Freiwillige Selbstverstümmelung. Warum eigentlich nicht?, in: Johann S. Ach, Arnd Pollmann (Hrsg.): no body is perfect. Baumaßnahmen am menschlichen Körper - Bioethische und ästhetische Aufrisse, Bielefeld 2006, S. 163-184 
Skatssoon (2005): Judy Skatssoon, The ethics of amputation by choice, ABC Science Online, News in Science, www.abc.net.au/science/news/stories/s1395891.htm, 21.06.2005 [06.02.2007]

Smith/Fisher (2003): Robert Smith, Keren Fisher, Letter to the editor: Healthy limb amputation: ethical and legal aspects, Clinical Medicine 3 (2003), 2, p. 188

Sorene et al. (2006): Elliot D. Sorene, Carlos Heras-Palou, Frank D. Burke, Self-amputation of a healthy hand: a case of body integrity identity disorder, Journal of Hand Surgery (British and European Volume), 31B (2006), 6, p. 593-595

Sosis (2005): Richard Sosis, Teure Rituale, Gehirn \& Geist 1-2 (2005), S. 44-50

SPIEGEL Online, Designer-Vagina: Warnung vor dem Schnitt im Schritt, 25.05.2007, www.spiegel.de/ wissenschaft/mensch/0,1518,484816,00.html

Steinmetzer/Groß (2007): Jan Steinmetzer, Dominik Groß, Transsexualität - Rechtliche und ethische Implikationen, in: B. Sharon Byrd, Jan C. Joerden (Hrsg.), Jahrbuch für Recht und Ethik, Band 14, 2006/07, S. 581-609

Storm/Weiss (2003): S. Storm, Michael D. Weiss, Self-inflicted tourniquet paralysis mimicking acute demyelinating polyneuropathy, Muscle \& Nerve 27 (2003), p. 631-635

Tammer (2006): Rona Tammer, Genezen van een dwangneurose. ,Ik sopte zelfs de stofzuiger', Psychologie Magazine (2006), p. 22-25

The Independent (2002): Disturbed Patients have healty limbs amputated, The Independent, London, 01.02.2002

Tomasini (2006): Floris Tomasini, Exploring ethical justification for self-demand amputation, Ethics \& Medicine 22 (2006), 2, p. 99-115

Wallace (2005): George F. Wallace, Indications for amputations, Clinics in Podiatric Medicine and Surgery 22 (2005), p. 315-328

Wise/Kalyanam (2000): Thomas N. Wise, Ram Chandran Kalyanam, Amputee fetishism and genital mutilation: case report and literature review, Journal of Sex \& Marital Therapy 26 (2000), p. 339-344

Witt et al. (2006): Karsten Witt, Christine Daniels, Jan Herzog et al., Differential Effects of L-Dopa and Subthalamic Stimulation on Depressive Symptoms and Hedonic Tone in Parkinson's Disease, Journal of Neuropsychiatry and Clinical Neurosciences 18, August (2006), p. 397-401

World Health Organization (1992): World Health Organization (ed.), The ICD-10 Classification of Mental and Behavioural Disorders. Clinical Descriptions and Diagnosic Guidelines, Genua 1992

Zimbardo (1995): Paul Zimbardo, Psychologie, Berlin, Heidelberg, New York, 6. Aufl., 1995

\section{Internet}

http://de.wikipedia.org (Wikipedia)

http://lists.ruhr-uni-bochum.de/mailman/listinfo/medethik-list (Mailingliste Medethik-list)

www.amputee-online.com (Amputee Web Site)

www.biid.org (Body Integrity Identity Disorder)

www.chirurgie-portal.de/orthopaedie/arm-bein-amputation.html (Chirurgie Portal)

www.transabled.org/ (Transabled.org) 